\title{
EXPERIMENTANDO CON ARMAS IBÉRICAS DE HIERRO. LA PRODUCCIÓN DEL METAL EN HORNOS DE "TIRO NATURAL"
}

\author{
EXPERIMENTING WITH IBERIAN IRON WEAPONS. \\ THE PRODUCTION OF METAL IN NATURAL DRAFT FURNACES
}

POR

\author{
José Miguel Gallego Cañamero*
}

“(Los íberos) Tienen un modo singular de preparar las armas que utilizan para su defensa. Entierran láminas de hierro y las dejan hasta que, con el tiempo, la parte débil del hierro consumida por la herrumbre se separa de la parte más dura; de ésta hacen espadas excelentes y los demás objetos concernientes a la guerra". Diodoro de Sicília, Bibliotheca Historica, I, 329

\section{Resumen - Abstract}

Se exponen los resultados de dos intentos experimentales para obtener hierro en sendos hornos del tipo de "tiro natural" mediante el proceso de reducción directa, extrapolando sus posibilidades para el mundo ibérico. Se describen los principios físicos que intervienen en el proceso y se propone un modelo de explotación minero- metalúrgica basado en la concentración de los medios de producción en zonas próximas a las materias primas (mineral y madera).

We present the results of two experimental attempts to obtain iron in natural draft furnaces by bloomery process, extrapolating its possibilities for the Iberian cultures. We describe the physical processes involved in the process and we propose a model of metallurgical and mining exploitation based on the concentration of the means of production in areas closed to raw materials (ore and wood).

\section{Palabras Clave - Keywords}

Arqueología experimental; hierro; armamento ibérico.

Experimental archaeology; bloomery process; natural draft furnaces; Iberian weaponry.

\section{INTRODUCCIÓN}

La información que en la actualidad poseemos sobre el armamento de hierro de los pueblos de la antigua Península Ibérica proviene de abundantes estudios que, durante años, han venido realizando diferentes investigadores, publicados en ocasiones con carácter monográfico. Entre ellos hay que destacar, obligatoriamente, las constantes aportaciones del profesor

\footnotetext{
*ARTIFEX., artifex.crpa@gmail.com.
} 
Quesada, y principalmente su tesis doctoral (Quesada, 1997) herramienta fundamental para el conocimiento de las armas proto-históricas peninsulares, aunque recientemente, jóvenes investigadores han iniciado excelentes proyectos de investigación que han visto la luz en forma de tesis doctorales (Garcia, 2006 y 2012) y que amplían notablemente nuestros puntos de vista. Existen, por otro lado, algunos estudios basados en las analíticas mediante microscopios electrónicos de barrido (SEM por sus siglas en inglés) de los hierros protohistóricos cuyo objetivo es el conocimiento estructural y la calidad de los metales (Auladell, 2004). Sin embargo y por lo general, todos estos estudios han quedado circunscritos al ámbito del análisis desde perspectivas teóricas principalmente de carácter morfológico, tipológico, funcional y simbólico o desde el punto de vista químico, obviando normalmente aspectos de contenido empírico.

Queda, por tanto, en la historiografía sobre las armas de hierro de los pueblos pre-romanos de la Península Ibérica, un terreno sin cultivar que puede llegar a ofrecer una perspectiva adicional a determinadas cuestiones, a nuestro juicio, sumamente importantes para conocer los métodos de fabricación de las armas: ¿enterraban los artesanos ibéricos láminas de hierro para eliminar determinados óxidos como citan algunos autores clásicos?; ¿tenía ello realmente una utilidad práctica?; ¿controlaban el ennegrecimiento con magnetita de las superficies de armas como la falcata?; ¿empleaban en la producción de hierro la técnica del "tiro libre" tanto como la de "alimentación asistida"?. Éstas, entre muchas otras, son cuestiones que aún permanecen sin resolver y que oscurecen el panorama tanto en lo que respecta al proceso de obtención del hierro como a su posterior transformación en herramientas bélicas.

Ésta es, en esencia, la línea de investigación que pretendíamos continuar con el presente artículo, el cual representa el segundo paso de un proyecto mucho más ambicioso que iniciamos en 2009. Como ya habíamos señalado en un trabajo anterior (Gallego, 2013), en su desarrollo preveemos la reproducción experimental de toda la cadena de obtención de hierro y su posterior transformación en armas durante el periodo ibérico, produciendo esponjas de hierro en hornos de reducción de diferentes tipologías, su cotejación estructural con piezas arqueológicas originales (siempre que sea posible) y su posterior empleo y manipulación. Es nuestra intención llegar a establecer una serie de matrices con las que establecer posibles pautas de fabricación, procedencia de la materia prima o utilización de posibles elementos fundentes, entre otras cosas, algo que sólo podremos conseguir a través del análisis comparativo de una cantidad importante de material, tanto original como reproducido. Sólo es posible que podamos contribuir a un conocimiento más específico de las herramientas bélicas de los pueblos ibéricos si consiguiéramos llegar a reproducir las etapas procesales que dieron lugar a los objetos originales, recreando las mismas peculiaridades físicas y técnicas. En este sentido, el presente artículo propone una perspectiva alternativa sobre las fases iniciales de la producción del armamento ibérico de hierro, determinantes para conocer sus peculiaridades tecnológicas pero también para establecer aproximaciones derivadas de su fabricación que resulten plausibles hacia su valor como objeto, y por ende, como símbolo en las sociedades ibéricas.

\section{ANTECEDENTES}

Los estudios específicos sobre los métodos de producción siderúrgica entre los pueblos de la protohistoria de la Península Ibérica no son muy abundantes en la historiografía española. Si nos ceñimos a una óptica experimental, esta carencia es del todo aplastante, muy al contrario de lo que ocurre en otros países europeos donde esta rama de la arqueología goza de una excelente salud (las aportaciones de Andrieux, Crew, Domergue, Dubois o Tylecote, sólo por citar algunos ejemplos, son dignas de mención). Desgraciadamente, tampoco podemos mencionar 
la existencia en España de estudios en los que la producción experimental de hierro ibérico se haya concebido como un sub-apartado dentro de un análisis más complejo, que contemple el proceso integral de elaboración de armas de hierro durante la Antigüedad, desde la obtención del mineral en la mina a cielo abierto hasta el trabajo del metal en los talleres de fragua, tan abundantes en los asentamientos pre-romanos.

Las primeras reducciones experimentales de mineral de hierro llevadas a cabo en la Península Ibérica, aunque sin adscripción arqueológica, fueron organizadas por el Museu d'Història de l'Hospitalet de Llobregat (Barcelona), en el año 1990. Un año más tarde, en octubre de 1991, fue el Govern d'Andorra el organizador de un evento similar. En ambos casos, el director técnico fue el maestro Phillippe Andrieux y también, en ambos, colaboraron estudiantes universitarios en la construcción de las estructuras fornarias y en la inyección de aire mediante fuelles. Desgraciadamente, ninguna de estas experiencias fue nunca publicada ${ }^{1}$.

No fue hasta quince años después, durante el año 2005, cuando el profesor S. Rovira retomó aquellos pasos, con el desarrollo de una serie de experimentaciones en el yacimiento celtibérico de Segeda, en el marco de un curso de arqueología experimental para estudiantes del que nosotros mismos fuimos testigos directos, y que fue publicado inmediatamente (Burillo y Rovira, 2005). Efectivamente, la documentación en dicho yacimiento de los restos de un bajo horno siderúrgico de reducción directa con fosa para las escorias, permitió la construcción experimental de varias interpretaciones de la estructura original. En el año 2005, y con la colaboración de algunos estudiantes, el profesor Rovira dirigió tres intentos de reducción de mineral de hierro (hematites, para ser exactos), que culminaron con la obtención de "[...] una escoria con abundante hierro metálico [...] de poco rendimiento [...]" (Rovira et alii, 2012: 30).

Finalmente, en el año 2009, la empresa ARTIFEX, conservación y recreación del patrimonio arqueológico de la cual formamos parte, inició un proceso de investigación siderúrgica durante época ibérica que culminó con la construcción de tres hornos de alimentación asistida, el primero de los cuales se construyó en colaboración con el grupo de recreación histórica Ibercalafell, que produjeron diferentes resultados (Gallego, 2013).

Excluyendo las aportaciones ya citadas y las que presentaremos aquí, no podemos mencionar ninguna actividad adicional desarrollada para conocer cómo los antiguos pueblos de la Península Ibérica obtenían hierro a partir de los minerales, al menos con constancia en lo que a bibliografía se refiere. En este sentido, no podemos aceptar como experimentación científica aquellas actividades que se llevan a cabo utilizando técnicas, elementos y medios modernos, por mucho que aparezcan en congresos de arqueología experimental. Pensamos que obtener hierro utilizando ventiladores eléctricos, elementos fundentes químicos o combustibles modificados industrialmente para obtener un mayor rendimiento calorífico, no puede ser nunca considerado como una actividad de experimentación científica, más aún cuando, el objetivo de las mismas no es responder las cuestiones que las evidencias arqueológicas plantean constantemente. Siguiendo la filosofía del profesor Ph. Andrieux (Manjin, 2004: 10), a menudo se confunde el concepto de experimentación arqueológica con el "vamos a ver qué pasa". Creemos firmemente que la disciplina de la arqueología experimental debe ser, en todo lo posible, absolutamente rigurosa y fiel a los datos arqueológicos disponibles $\mathrm{y}$, además, debe contemplar necesariamente el estudio y conocimiento de los elementos periféricos al objeto de la experimentación. En el caso de la siderúrgia ibérica, esto implica la elaboración del combustible, la obtención del mineral, el trabajo del metal resultante, la elaboración de armas y su posterior testeo, pero también la experimentación con láminas y esponjas de hierro siguiendo las indicaciones de algunos clásicos que hacen referencia a la preparación de armas por parte

1 (Rovira, C., com. pers.). 
de los pueblos peninsulares, como la que encabeza este artículo. Evidentemente, la experimentación científica debe tener como finalidad el registro minucioso de la mayor cantidad de datos posible y la corrección de los errores cometidos anteriormente. Sólo partiendo de las bases documentadas que nos proporcionan los indicios reales y físicos, podremos plantear interpretaciones fiables y ópticas útiles a la comunidad científica.

Dejando de lado los restos de hornos de reducción en contextos coloniales fenicios, existen evidencias de hornos de reducción directa en un buen número de asentamientos ibéricos desde Alicante a Catalunya, datados entre el s. VII, en el caso más antiguo (Els Vilars) y el II a. C. en los casos más modernos (El Camp de les Lloses), aunque en su mayor parte se trata de estructuras fechables a partir del s. IV a. C. correspondientes al tipo "con fosa para escorias". Sin embargo, actualmente desconocemos todavía muchos de los detalles técnicos y funcionales asociados a estas estructuras que los "herreros" ibéricos debían controlar. En este sentido, si en el registro arqueológico ya resulta difícil distinguir restos de hornos de reducción directa de restos de pequeñas fraguas, aún es más difícil afirmar si se trata de estructuras de combustión asistidas mediante fuelles o si se trata de estructuras auto-alimentadas o de "tiro libre". Normalmente se ha tendido a pensar en la presencia de fuelles para insuflar aire al interior del horno, incluso en aquellos casos en los que no existía ninguna evidencia arqueológica que apuntase en esa dirección, quizá por tratarse de la opción más recurrente, descartando a priori la posibilidad de que se tratase de hornos de tiro libre. A nuestro juicio y como explicaremos más adelante, el diámetro de las cubetas de combustión podría constituir un elemento determinante a este respecto, dado que guarda una relación con la altura total del horno.

Si bien es cierto que se ha podido comprobar experimental y etnográficamente la funcionalidad de los hornos de reducción directa asistidos mediante fuelles, con el presente artículo pretendemos matizar esta posibilidad en el mundo ibérico.

\section{DESCRIPCIÓN DE LOS HORNOS DE REDUCCIÓN DIRECTA}

Como ya expusimos en un trabajo anterior (Gallego, 2013), técnicamente, un horno es una estructura de combustión en cuyo interior se someten materiales a temperaturas elevadas. En función del uso que se le vaya a dar y de la temperatura que se pretenda alcanzar, los hornos deben construirse con unas determinadas características que permitan distribuir lo más homogéneamente posible el calor en su interior (Rehder, 2000: II, 14). Los hornos de reducción directa de hierro documentados en contextos arqueológicos prerromanos de la Península suelen ser estructuras construidas generalmente con arcillas y piedras y elementos orgánicos vegetales (según ha desvelado el registro arqueológico (Morer y Rigo, 1999; Rovira y Burillo, 2005), probablemente con forma cilíndrica o cónica ${ }^{2}$, más amplios y voluminosos en la parte inferior (cubeta) y más estrechos en la mitad superior (chimenea), en cuyo interior se somete el mineral de hierro (óxidos e hidróxidos) a altas temperaturas en una atmósfera rica en el carbono liberado por el carbón para separar el hierro puro $(\mathrm{Fe})$ del resto de componentes del mineral. En el caso de los hornos de "tiro natural" o "tiro libre", es el propio horno el que toma el aire del exterior a través de las toberas, siguiendo un principio de física básica que veremos más

2 Por lo general, en el registro arqueológico no se conserva la parte aérea de éstas estructuras. Lo habitual es encontrar un negativo, con las paredes rubefactadas con diferente intensidad, a veces conteniendo carbones, cenizas y material escoriáceo. En ocasiones, esto ha hecho pensar a algunos autores que el mineral de hierro era reducido en un simple agujero excavado en el suelo con la asistencia de fuelles (Morer, com. pers.), conclusión, a nuestro juicio, no siempre correcta si tenemos en cuenta la escasa rentabilidad incluso de estructuras más voluminosas y consideradas, por tanto, más productivas (vid. infra, 18-19). 
adelante. Este sistema ha sido empleado sistemáticamente en algunas zonas de África hasta la actualidad y ha podido ser constatado arqueológicamente en diferentes contextos geográficos y cronológicos (Martinelli, 2008; Serneels et alii, 1997).

Los bajos hornos de reducción directa pueden ser del tipo "con fosa para escorias" o "con canal de drenaje de escorias", excavada ex professo por debajo de la cubeta, o que las escorias son evacuadas mediante una apertura en la parte baja del horno, respectivamente. No obstante, en el mundo ibérico no se conservan evidencias del segundo tipo, pudiendo tratarse de una innovación introducida durante época romana. Para su correcto funcionamiento, el horno debe pre-calentarse durante algún tiempo, en función de diversas variables como el grado de humedad de la estructura, la altura o la temperatura y la humedad ambientales

Para obtener hierro, hay que someter el mineral a una reacción química, denominada "reducción directa", a la que sólo se puede acceder mediante temperaturas muy elevadas. A partir de los $700{ }^{\circ} \mathrm{C}$, los óxidos e hidróxidos de hierro empiezan a reaccionar con el monóxido de carbono $(\mathrm{CO})$ que desprende el carbón vegetal, liberando dióxido de carbono $\left(\mathrm{CO}_{2}\right)$ y aislando el hierro puro $(\mathrm{Fe})$ tal como se describe en la fig. 1 :

Esta temperatura de inicio de la reducción es aún muy inferior a la temperatura que se debe alcanzar para que los otros componentes de la mena se separen molecularmente del metal, del orden de los $1.100{ }^{\circ} \mathrm{C}-1.200{ }^{\circ} \mathrm{C}$, aproximadamente. Cuando se alcanza este punto, el hierro se separa químicamente del resto de elementos presentes en el mineral dando lugar a una masa informe (conocida también como lupia) en la que se encuentran mezclados el hierro puro y las escorias de hierro, que no son otra cosa que material residual fundido o semi-fundido, que será necesario separar físicamente en la fragua y el yunque.

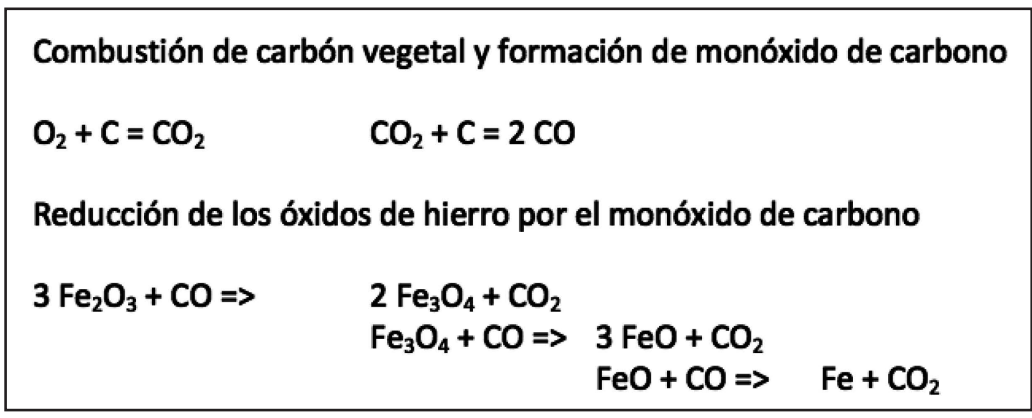

Figura 1. Secuencia de la transformación de los óxidos e hidróxidos de hierro mediante el proceso de reducción directa (extraído de Leroy, M., Merluzzo, P., 2004: 2, 49).

\section{LOS HORNOS EXPERIMENTALES}

Ambos hornos fueron construidos en una suave pendiente de Serra de Les Martines (Terrassa, Barcelona), perteneciente a la Cordillera Pre-litoral, abierta hacia el gran plano de la comarca del Vallès Occidental con orientación hacia el N (UTM: X417821.0/ Y4599407.3).

3 La clasificación tipológica de estos hornos suele conllevar cierta complejidad, dado que en el registro arqueológico normalmente sólo se conservan los elementos excavados, perdiéndose toda o casi toda evidencia de la parte aérea. Cleere y Forbes propusieron una clasificación para este tipo de estructuras, basándose en las características morfológicas de las mismas (Cleere, 1972; Forbes, 1972). 


\section{HORNo 1: ELTERKER (fig. 2)}

Su singularidad reside en el hecho de que es el primer horno de estas características construido experimentalmente en la Península Ibérica, hasta donde hemos logrado averiguar. Pensamos que este tipo de hornos fueron completamente funcionales y más adecuados para la transformación intensiva del mineral a pie de mina durante época ibérica que los asistidos manualmente, en línea con las evidencias que se han podido definir en otros centros de pro-

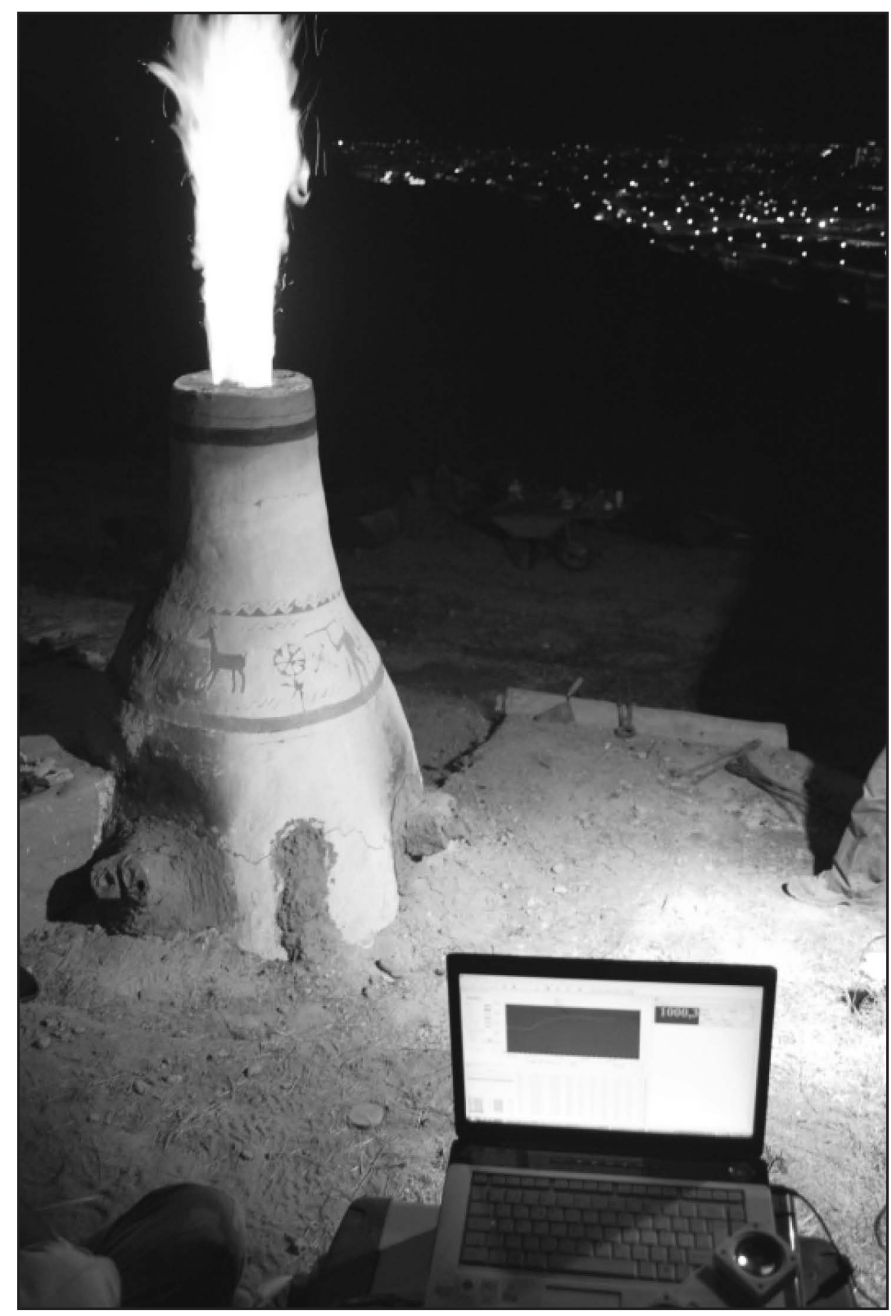

Figura 2. El horno experimental de tiro libre "ELTERKER" (Foto: ARTIFEX, crpa).

ducción galos y galo-romanos sincrónicos (Dunikowski y Cabboi, 1995; Decombeix et alii, 2001). Como tendremos oportunidad de describir más adelante, presentan una serie de ventajas sobre los hornos alimentados mediante fuelles.

Con unas dimensiones mayores a los ejemplos asistidos con fuelles que ya estudiamos (Gallego, 2013), las reacciones químicas y los procesos físicos que intervienen en el proceso 
de reducción se ralentizan, por lo que la duración se multiplica por tres o cuatro, llegando a superar las 40 horas (en el caso que nos ocupa, fueron 42 exactamente). El horno en cuestión medía 1'80 m. de altura, con un diámetro interno de cubeta de 0'80 m. y un diámetro interno de chimenea de 0’25 m. Sobre una pequeña fosa para las escorias $\left(0^{\prime} 15 \mathrm{~m}\right.$.), se construyó la estructura fornaria utilizando una mezcla de tierra arcillosa con inclusiones de nódulos calcáreos de pequeñas dimensiones procedente de depósitos miocénicos próximos (85\%), piedras de tipo calcáreo y sedimentario de procedencia local $(10 \%)$ y fibras vegetales de trigo (Triticum Dicocum) (5\%). Como en los ejemplos anteriores, la pared interna fue recubierta con una capa de 1'5 cm. (aproximadamente) de material refractario preparado a partir de arcillas de origen local depuradas mediante tamizado y refinadas mediante flotación (85\%), estiércol de caballo (Equus Ferus Caballus) (10\%) y cenizas de olivo (Olea Europaea) (5\%). Esta capa, además de evitar posibles fisuras provocadas por las altas temperaturas, uniformiza y homogeniza la pared interior, anulando aristas y evitando la retención de material en su descenso hacia la cubeta de combustión.

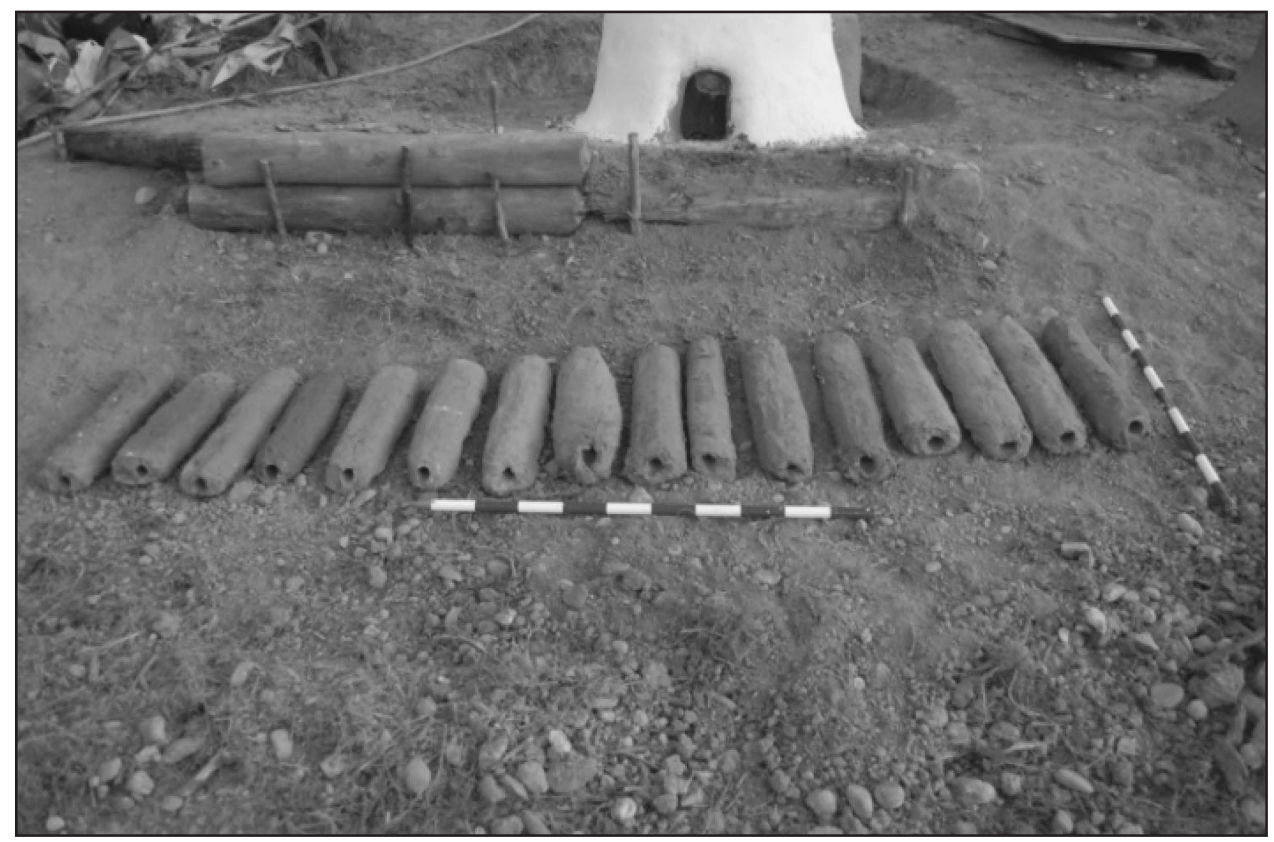

Figura 3. Toberas cilíndricas secándose al sol (Foto: ARTIFEX, crpa).

Encendido en precalentamiento desde las 18:45 h. hasta las 21:45 h., en la parte inferior se prepararon cuatro aberturas orientadas hacia los cuatro puntos cardinales para la ubicación de las toberas y para poder introducir herramientas con las que acceder, una vez abierto, a las posibles esponjas. Para cerrarlo, primeramente se colocaron trece toberas con una longitud media de $50 \mathrm{~cm}$. y un conducto de $2^{\prime} 5 \mathrm{~cm}$. de diámetro con una inclinación de unos $30^{\circ}$, entrando $12-15 \mathrm{~cm}$. en el interior del horno y a unos $20 \mathrm{~cm}$. de la base de la cubeta que habían sido elaboradas previamente y dejadas para su secado natural (fig. 3).

Los espacios que quedaban entre las toberas se taponaron con una mezcla de barro y paja de trigo (Triticum Dicocum) para evitar una entrada de aire incontrolada. A lo largo del proceso 


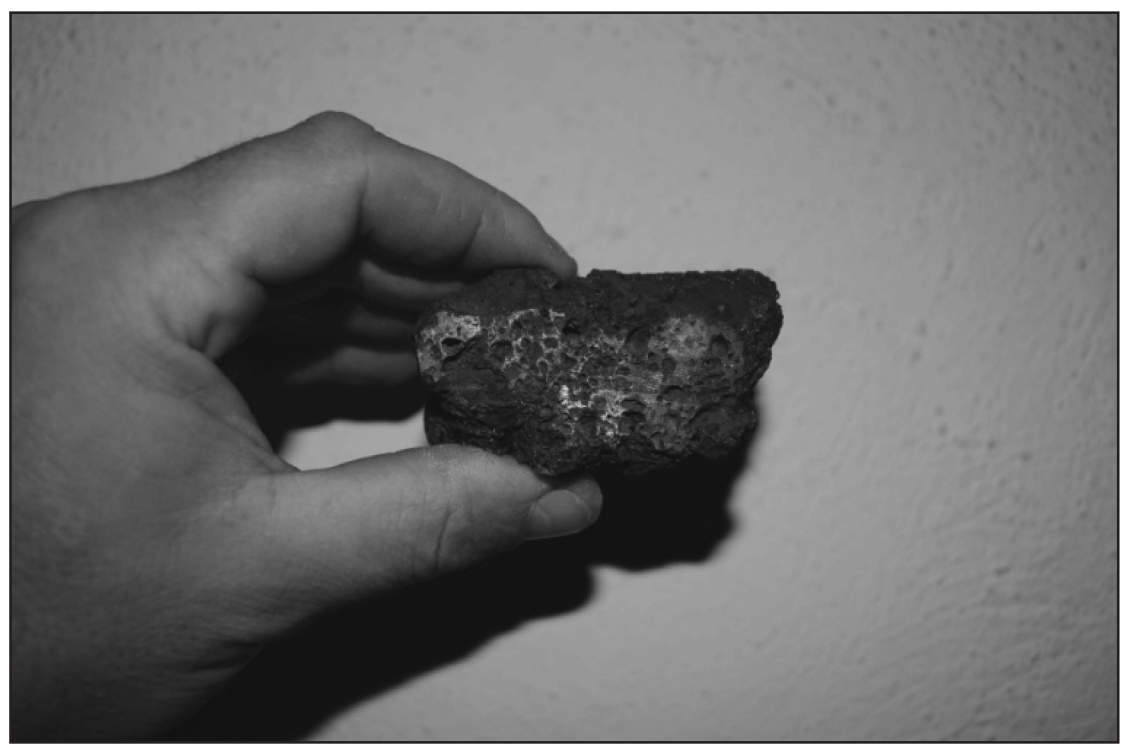

Figura 4. Uno de los fragmentos seccionados de una esponja de hierro del horno "ELTERKER" (Foto: ARTIFEX, crpa)

de reducción, el horno se cargó con ciclos $^{4}$ alternativos que fueron introducidos por la parte superior de la chimenea hasta alcanzar $100 \mathrm{~kg}$. de carbón vegetal de olivo (Olea Europaea) y acebuche (Olea Europaea Sylvestris) en una proporción del 60\% y del 40\% respectivamente, y $31^{\prime} 5 \mathrm{~kg}$. de mineral sin tostar del tamaño de una nuez (limonita con goethita y hematita procedente de la mina de Rocabruna, en Gavà), en una ratio de 3'1 kg. de carbón por cada uno de mineral. Finalmente, se retiraron las toberas, se liberaron las aperturas y se vació el contenido del horno. La temperatura fue controlada mediante un data logger modelo YC-747UD de la marca YCT y un termopar 14- S- I- 6- IRA- 300 (90\% platino, $10 \%$ rodio recubierto de porcelana refractaira) de la marca TC medida y control de temperatura, $S$. A. que fue ubicado entre dos de las zonas donde se habían insertado la toberas, a la misma altura que éstas, entrando unos $20 \mathrm{~cm}$. en el interior del horno. Se alcanzó un registro medio sobradamente por encima de $\operatorname{los} 800{ }^{\circ} \mathrm{C}$ y se mantuvo por encima de $\operatorname{los} 1000^{\circ} \mathrm{C}$ (alcanzando un techo de $1017^{\circ} \mathrm{C}$ ) durante casi una hora, lo que produjo en el interior del horno unos $10 \mathrm{~kg}$. de lupias de diversas dimensiones en estado semi-viscoso alrededor de la boca de las toberas, como es sabido, la zona de mayor incidencia térmica por tratarse de las entradas de aire, que fueron extraídas tras poco más de 50 horas de combustión. Una parte de este material está en el momento de redación de éstas líneas pendiente de estudio, aunque tras un análisis macroscópico podemos confirmar la presencia de pequeños núcleos de hierro puro (fig. 4).

\section{Horno 2: Elkinke (fig. 5)}

Aunque este horno aprovechaba la estructura del anterior, dado que apenas había sufrido alteraciones derivadas de las altas temperaturas alcanzadas, el proceso de reducción sufrió algunas modificaciones estructurales y periféricas que veremos a continuación.

4 Un ciclo es una secuencia definida por una carga de carbón seguida de una de mineral. 
Para empezar, los $110 \mathrm{~kg}$. de mineral (limonita con goethita y hematita) fueron "enriquecidos", mediante su tostado (fig. 6), previamente a su introducción en el horno. El enriquecimiento del mineral ha podido ser documentado en diferentes yacimientos arqueológicos de la Primera y Segunda Edad del Hierro, peninsular y europea (como Les Guàrdies en el Vendrell (Morer y Rigo, 1999) y Clerimois en el Yonne francés (Dunikowski y Cabboi, 1995), sólo por

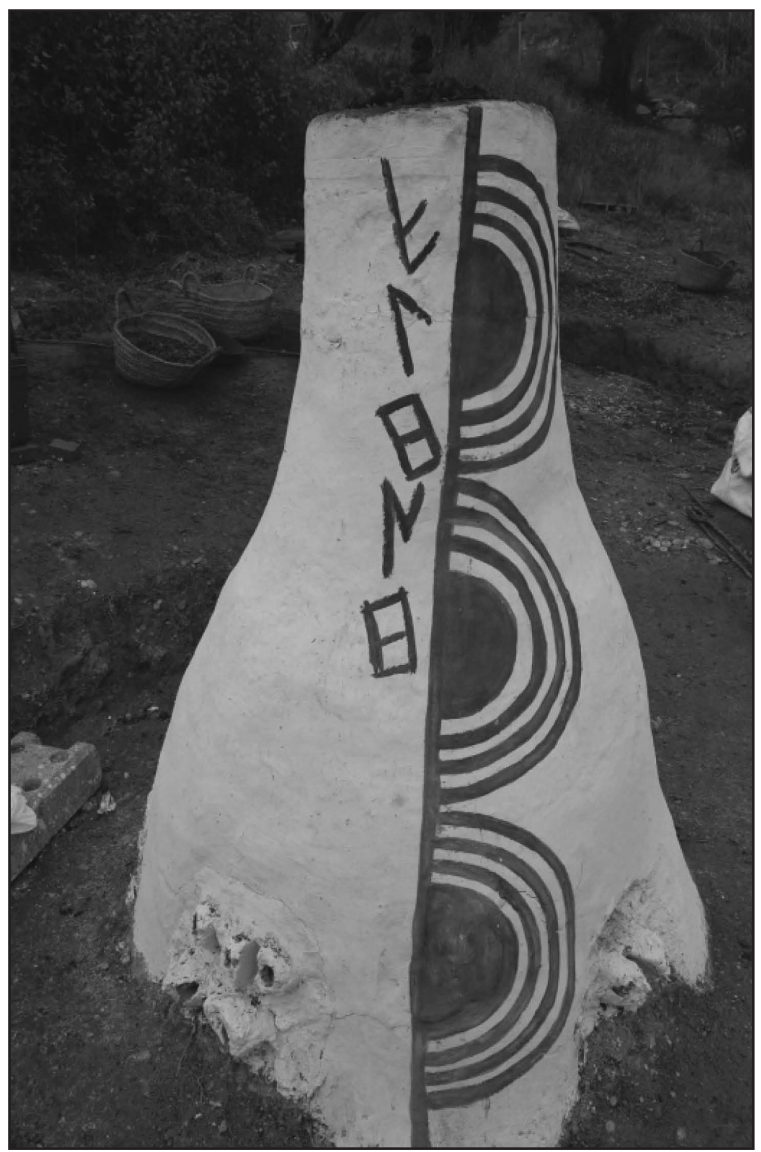

Figura 5. El horno experimental de tiro libre "ELKINKE" (Foto: ARTIFEX, crpa).

citar dos ejemplos. Consiste en someter el mineral a altas temperaturas durante algunas horas para alterar su composición física y química y así deshidratarlo y hacerlo fragmentable. Tras esta operación, el mineral fue reducido a un tamaño menor (módulos de $1^{\prime} 5 \mathrm{~cm}$. aproximdamente) que en el horno anterior, propiciando así la futura acción de los gases reductores en el interior del horno de reducción. Tras el tostado, el peso del mineral ya deshidratado quedó ligeramente reducido a $100 \mathrm{~kg}$., lo que representa la pérdida de menos de un $10 \%$ del peso original.

El sistema de respiración mediante toberas de tiro libre también sufrió algunas modificaciones. En lugar de las trece que se colocarón en el anterior horno de tiro libre, en esta ocasión se colocaron quince, aprovechando los espacios dejados para tal propósito en las paredes del horno, y conservando, por tanto, la misma orientación que en caso anterior. Su fabricación se 


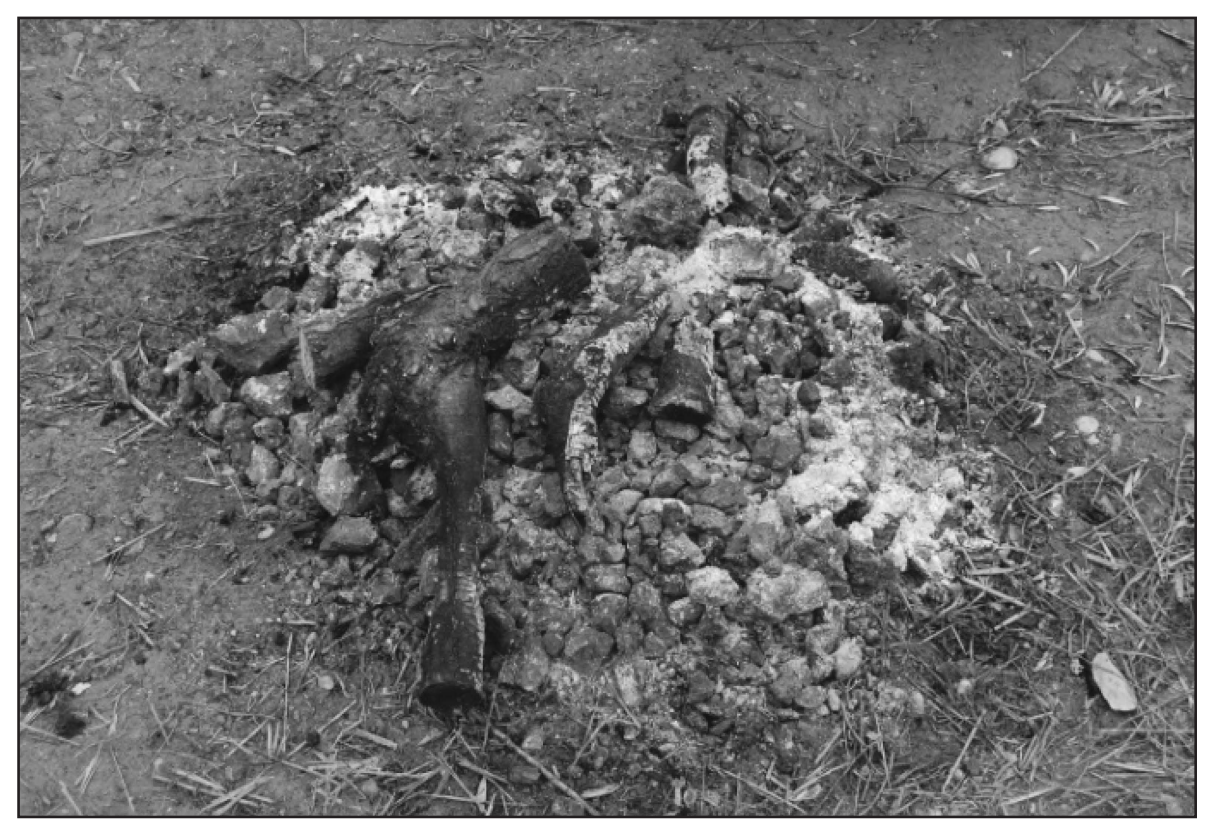

Figura 6. Hoguera de enriquecimiento o deshidratación tras la combustión (Foto: ARTIFEX, crpa).

llevó a cabo utilizando exactamente la misma materia prima que en el hono anterior y respetando las mismas proporciones. Sin embargo, fuimos más cuidadosos con los acabados de las mismas, que experimentaron una mejora morfológica respecto a sus predecesoras. Se utilizó la mitad de masa para la elaboración de cada una, con lo que conseguimos unas toberas mucho más delgadas, aun manteniendo el mismo diámetro interno de 2'5 cm.

Encendido en precalentamiento con madera seca de pino blanco (Pinus Halepensis) a las 18:05 h., se mantuvo en combustión hasta las 20:40 h. momento en el que se colocaron las toberas de ventilación. Como combustible, volvimos a emplear carbón de olivo (Olea Europaea). Para obtener $85 \mathrm{~kg}$. fue necesario combustionar en la carbonera $900 \mathrm{~kg}$. de madera, lo que significa una rentabilidad apenas del $10{ }^{\prime} 5 \%^{5}$. En esta ocasión, la ratio fue de 0 ' $85 \mathrm{~kg}$. de carbón por cada uno de mineral algo que, como veremos posteriormente, se encuentra muy por debajo de los mínimos necesarios para una reducción exitosa estudiados por algunos autores (Rehder 2000). La temperatura media sobrepasó los $800^{\circ} \mathrm{C}$ y un techo de $1095^{\circ} \mathrm{C}$ durante prolongados periodos de tiempo, aunque a lo largo de la experimentación se produjeron tres picos de más de $1000{ }^{\circ} \mathrm{C}$ durante periodos de aproximadamente una hora. Finalmente, el horno se abrió después de 88 horas. Conseguimos aproximadamente $20 \mathrm{~kg}$ de material escoriáceo con evidentes zonas en estado viscoso. Macroscópicamente, éste material parece indicar una reducción incompleta, con abundante presencia de nódulos de mineral semi-fundido. Desgraciadamente, en este caso también estamos pendientes de su análisis.

\footnotetext{
5 La elección de la madera de olivo o acebuche (Olea Europaea Sylvestris) se debe, primeramente, a su presencia en el yacimiento ibérico de Les Guàrdies (Morer y Rigo, 1999), pero también a sus características naturales. Se trata de una madera de muy alta densidad (0’98 g/cm3) cuyo carbón ofrece una de las mayores capacidades caloríficas (entre 31.000 y $16.720 \mathrm{kj} / \mathrm{kg}$.). Si tenemos presente que para producir $85 \mathrm{~kg}$. de este carbón, hicieron falta $900 \mathrm{~kg}$. de madera verde, apreciaremos enseguida la cantidad de árboles que harian falta para mantener una producción de hierro relativamente intensa, de ahí los argumentos para emplazar estos centros en las proximidades de las zonas ricas en recursos. En nuestros objetivos también figura el estudio de la producción artesanal de carbón para la elaboración de armas de hierro durante época ibérica, tema que pretendemos abordar en el futuro.
} 


\section{DISCUSIÓN}

Aunque somos plenamente conscientes de que dos ejercicios de experimentación con este tipo de hornos no suponen una base cuantitativa demasiado amplia sobre la que plantear conclusiones determinantes, sí que definen una plataforma empírica lo suficientemente sólida como para permitirnos plantear algunas hipótesis iniciales sobre los sistemas de producción de hierro durante la Protohistoria de la Península Ibérica, por un lado, y acercarnos a la comprensión de los complejos elementos que intervienen en los procesos de reducción, por el otro. Adicionalmente, un análisis microscópico en profundidad de los materiales obtenidos y de los minerales empleados en las reducciones descritas podría ofrecernos un punto de vista complementario.

Contamos con abundante información arqueológica sobre el proceso siderúrgico entre los ss. IV al II a. C., recuperada en diversos asentamientos ibéricos, desde la extracción del mineral (Álvarez y Estrada, 2009) hasta la fabricación de utensilios de hierro (Auladell y Simon 1997; Auladell, 2004; Simon et alii, 2005), pasando por la reducción propiamente dicha (Morer y Rigo 1999), las escorias ferríferas (Gómez, 1996), o los elementos periféricos de depuración y forja (Molist, 2005) que nos puede servir para entender los procesos post-reducción y para establecer una primera aproximación hipotética sobre la materia.

Defenderemos a continuación la existencia en el mundo ibérico de determinados centros dedicados específicamente a la producción de hierro, a pie de mina, alejados de los centros urbanos, dirección hacia la que ya apuntaban los indicios exhumados en el yacimiento ibérico de les Guàrdies, anteriormente citado, o en el yacimiento ibérico de Mas d'en Gual (El Vendrell, Tarragona), argumento que a priori nos aproxima a las afirmaciones de algunos autores (Bonet y Vives, 2011: 114; Rehder, 20006) y nos aleja de otros (Gómez, 1996). A modo de resumen, consideramos plausible que en estos centros, los encargados de la producción de hierro ibéricos recurrieran exclusivamente a hornos de respiración libre, quedando los posibles hornos de reducción en poblado para producciones muy puntuales. Creemos firmemente que los hornos de alimentación asistida serían utilizados para llevar a cabo producciones muy limitadas y por las razones que expondremos a continuación, mientras que se utilizarían los hornos de tiro libre (incluso más de uno simultáneamente) para un tipo de producción mucho más intensiva y con una clara finalidad comercial en las proximidades de las explotaciones mineras o boscosas, como bien han apuntado ya diferentes autores (Mata, 2009).

No obstante, antes de entrar en detalles, estimamos necesario aclarar algunos principios relativos a la dinámica física y química de estas estructuras de combustión que hemos podido comprobar en nuestras experimentaciones.

Como ya se había expuesto anteriormente (Rehder, 2000) el principio básico que permite que un horno de estas características pueda alcanzar tan altas temperaturas (del orden de los $1100^{\circ} \mathrm{C}-1200^{\circ} \mathrm{C}$ ), no es una mayor cantidad de toberas o fuelles, ni el diámetro del conducto de aire de las toberas, ni siquiera la densidad y capacidad calorífica del carbón vegetal. En realidad, el efecto verdaderamente determinante en el proceso de reducción directa es la diferencia de presión existente entre el aire caliente del interior del horno y el aire a temperatura ambiente del exterior del mismo, y el efecto que en ello tiene la altura de la chimenea, como se refleja en la "ecuación de Bernoulli"?:

"[...] natural draft smelting furnaces were used more extensively in antiquity than is presently realized [...]" (Rehder, 2000: 128) y posteriormente insiste: "[...] in my opinion natural- draft charcoal fuelled shaft furnaces probably were used in anitiquity to a larger extent than is realized." (Rehder, 2000: 180).

7 Daniel Bernoulli (Groningen, 1700 - Basilea, 1786). En 1738, publicó Hydrodynamica, un trabajo que incluía la hidrostática y los sistemas hidráulicos, y que se basaba en el principio de la conservación de la energía cinética. En esta 


$$
\mathrm{V}^{2}(\mathrm{Di}-\mathrm{De}) / 2+\mathrm{P}+(\mathrm{Di}-\mathrm{De}) \mathrm{g} \mathrm{h}=\text { constante }^{8}
$$

Si observamos detenidamente la fórmula, veremos que el único factor que en realidad tiene un valor determinante es $h$, es decir, la altura del horno, lo que podemos traducir de la siguiente manera: a mayor altura del horno $(\mathrm{h})$, se produce una mayor succión de fluido (aire) a través de las toberas dado que el aire caliente asciende a lo largo de la chimenea como consecuencia de la diferencia de densidades entre el aire caliente del interior y del aire frío del exterior, resultando en el aumento de la cantidad de oxigeno en el interior del horno y, finalmente, en el incremento térmico.

Si a la ecuación anterior sumamos el Principio de Continuidad de los fluidos de la Dinámica de Fluidos se establece también que al reducir la sección transversal de un conducto por el que atraviesa un determinado volumen de fluido, aumentará su velocidad, como se ve en la siguiente ecuación ${ }^{9}$ :

$$
\mathrm{A}_{1} \cdot \mathrm{V}_{1}=\mathrm{A}_{2} \cdot \mathrm{V}_{2}
$$

Este dato explicaría porqué es conveniente que los hornos de reducción directa posean un orificio de evacuación en la chimenea de área menor que la de la cubeta de combustión, punto de origen del fluido. La gran llama que asoma por la boca de la chimenea del horno Elterker (fig. 2) responde a este principio físico.

Dicho de una forma más sencilla, el aire que entra en el horno a través de las toberas, tras calentarse en el interior de la cubeta, asciende con una densidad y peso específico menores que el del exterior ${ }^{10}$ que está más frio, provocando a su vez, la succión de aire a través de las toberas. La densidad del aire desciende a medida que aumenta la temperatura. Una chimenea alta, a través de la cual se precipite el aire caliente ascendente, provocará al mismo tiempo, una aceleración en la entrada natural de aire frio desde el exterior, lo que a su vez, acelerará el incremento de temperatura. No obstante, desconocemos aún la relación exacta entre el volumen y la velocidad de entrada de aire frio y el volumen y la velocidad de salida de gas caliente a través de la chimenea, pero pretendemos continuar avanzando en esta línea de investigación.

Para que la chimenea produzca ese efecto, su diámetro interno y su altura deben guardar una relación proporcional al diámetro interno de la cubeta, y a la altura total del horno, y por lo tanto a su volumen. Así, una cubeta muy voluminosa necesitará una chimenea muy alta lo suficientemente ancha como para que permita un flujo de aire caliente ascendente dinámico, que evacúe el volumen gaseoso generado en el interior del horno, y también para que la velocidad de entrada del aire a través de las toberas permita que se alcance el centro del horno. Igualmente, unas toberas con un conducto demasiado ancho provocarán un flujo de aire a baja velocidad que, probablemente, no alcanzará el centro de la campana del horno, dejando así una parte del material al margen de la reducción. En otras palabras, un diámetro de chimenea demasido ancho o demasiado estrecho, reducirán el volumen de salida de gases, que a su vez afectará negativamente al volumen y a la velocidad de entrada de aire frio, que consecuentemente reducirá la temperatura en el interior del horno, cosa que finalmente o bien provocará un aumento del tiempo necesario para producir la reacción química de la reducción o bien acabará asfixiando la combustión (en el peor de los casos). No obstante, aunque podemos afirmar que

\footnotetext{
obra se encuentra un primer borrador de la teoría cinética de los gases y un teorema, también llamado "de Bernoulli", que expresa la conservación de la energía mecánica en el flujo de un fluido perfecto.

8 Donde $\mathrm{V}=$ velocidad del aire, $\mathrm{Di}=$ Densidad del aire en el interior del horno, $\mathrm{De}=$ Densidad del aire en el exterior del horno, $\mathrm{P}=$ Presión a lo largo de la chimenea, $\mathrm{G}=$ fuerza de la gravedad (su valor es de 9' $80665 \mathrm{~m} / \mathrm{s}^{2}$ en la superficie de la Tierra, aunque generalmente se acepta el redondeo hasta $9^{\prime} 81 \mathrm{~m} / \mathrm{s}^{2}$ ) y H= altura total de la estructura.

$9 \quad \mathrm{~A}=$ área de la sección transversal del conducto, $\mathrm{V}=$ velocidad del fluido que atraviesa la sección.

10 La densidad del aire a $1.000^{\circ} \mathrm{C}$ es de $0^{\prime} 25 \mathrm{~kg} / \mathrm{m} 3$ mientras que a $20^{\circ} \mathrm{C}$ (temperatura ambiente) es de $1^{\prime} 25 \mathrm{~kg} / \mathrm{m} 3$.
} 
la altura de la chimenea debe ser, como mínimo, el mismo valor que el diámetro interno de la campana (vid. infra, 51), aún no hemos podido establecer con exactitud dicha proporción, la cual será objeto de estudio en futuras experimentaciones.

En cambio, como ya habíamos publicado anteriormente (Gallego, 2013), en el interior de un horno asistido con fuelles, el proceso natural de absorción de aire es controlado y acelerado por la corriente artificial inyectada mecánicamente, por lo que el resultado final no depende tanto de las características del horno sino, en definitiva, del correcto funcionamiento del sistema de fuelles y del tiempo que se dedique al ejercicio. Con este sistema, podría bastar con una pequeña fosa excavada en el suelo para reducir una pequeña cantidad de mineral, como algunos arqueólogos han propuesto para el caso del cobre (Forbes, 1971). No obstante, valdría la pena tener en cuenta los valores de mineral y carbón necesarios para obtener una cantidad suficiente de hierro con la que fabricar un simple clavo y si este sistema podría llegar a resultar rentable. Sirva como ilustración a esta afirmación la relación que sigue: Tylecote obtuvo una esponja de $8 \mathrm{~kg}$. a partir de $50 \mathrm{~kg}$. de mineral (hematita) y $40 \mathrm{~kg}$. de carbón, en 12 horas de reducción, con un horno de una sola tobera (Tylecote, 1971). Crew, en cambio, obtuvo una esponja de $100 \mathrm{~kg}$. a partir de $500 \mathrm{~kg}$. de mena (hematita) y $600 \mathrm{~kg}$. de carbón en un ciclo de 24 horas (Crew, 1998). Por su parte, Van de Mannaker obtuvo algo más de $23 \mathrm{~kg}$. de lupia en un primer horno experimental a partir de $90 \mathrm{~kg}$. de mena y $90 \mathrm{~kg}$. de carbón alimentado durante 14 horas. En el segundo horno, obtuvo $18^{\prime} 7 \mathrm{~kg}$. a partir de $45 \mathrm{~kg}$. de mena y $45 \mathrm{~kg}$. de carbón, inyectando aire durante 5'5 horas (Boonstra et alii, 1997). Una reducción más reciente en el horno de tiro libre Ingana, en el país Dogon en Mali, produjo unos $30 \mathrm{~kg}$. de esponja a partir de $200 \mathrm{~kg}$. de mena y de más de $250 \mathrm{~kg}$. de carbón (Serneels, com. pers.) Nosotros mismos obtuvimos una lupia de unos $10 \mathrm{~kg}$. a partir de $31^{\prime} 5 \mathrm{~kg}$. de mineral en el horno ELTERKER y de unos $22 \mathrm{~kg}$ a partir de $100 \mathrm{~kg}$. de mineral (deshidratado) en el horno ELKINKE. Sin embargo, las cantidades aquí descritas hacen referencia a la esponja ferrífera en bruto, la cual debe ser posteriormente depurada, perdiendo buena parte de su peso, normalmente entre un $80 \%$ y un $85 \%$. Como ejemplo de esta afirmación sirvan el primer horno de Van de Mannaker (Boonstra et alii, 1997) que produjo una esponja de 4'2 $\mathrm{kg}$. de la que finalmente se pudo obtener un lingote de 0 ' $7 \mathrm{~kg}$, esto es, una rentabilidad del $17 \%$, y el experimento de Dubois con uno de los ejemplares del conjunto de Lercoul que ofreció resultados inferiores (Dubois, $2000 \mathrm{y}$ especialmente 2001). La productividad final obtenida en los hornos de inyección asistida por Van de Mannaker y por Dubois puede ser extrapolable a los hornos de tiro natural, dado que la tipología del horno no es determinante en lo que a rendimiento se refiere, sino a los recursos necesarios para obtenerlo. O dicho de otro modo, un horno de tiro natural puede producir las mismas cantidades de metal que otro de inducción asistida pero invirtiendo menos esfuerzos. De ahí la opción de mantener una batería de ellos en funcionamiento simultáneo con los mismos recursos humanos que hacen falta para atender a uno sólo de inducción asistida.

Sabemos que las esponjas de hierro se forman justo en las proximidades de las entradas de aire, por ser las zonas más calientes de la estructura. La chimenea actúa únicamente como despensa deshidratante y como conducto de carga y salida de gases, y no alcanza temperaturas excesivamente altas (Andrieux, 2004: 70-71), por lo que no es un espacio útil donde producir metal férrico. Esa transformación tiene lugar en la cubeta junto a las entradas de aire que proporcionan las toberas. Teniendo en cuenta estas premisas, la cubeta de algunos de los hornos excavados en Les Guàrdies, cuyo diámetro interno conservado oscilaba en torno a $1 \mathrm{~m}$. (fig. 7), necesitarían de una chimenea con una altura mínima aproximada de 1m., si aceptamos la ratio mínima de 1/1 conservada en los hornos documentados en la Juncada de Sierra Menera (Fabré et alii, 2012: 43-62), en Clerimois (Dunikowsy y Cabboi, 1995) y en los hornos italianos de Populonia (Toscana) datados entre los ss. V y I a. C. (Gómez, 1996), (contando desde la base interna de la estructura o "solera"), de lo contrario, tendríamos simplemente una gran 


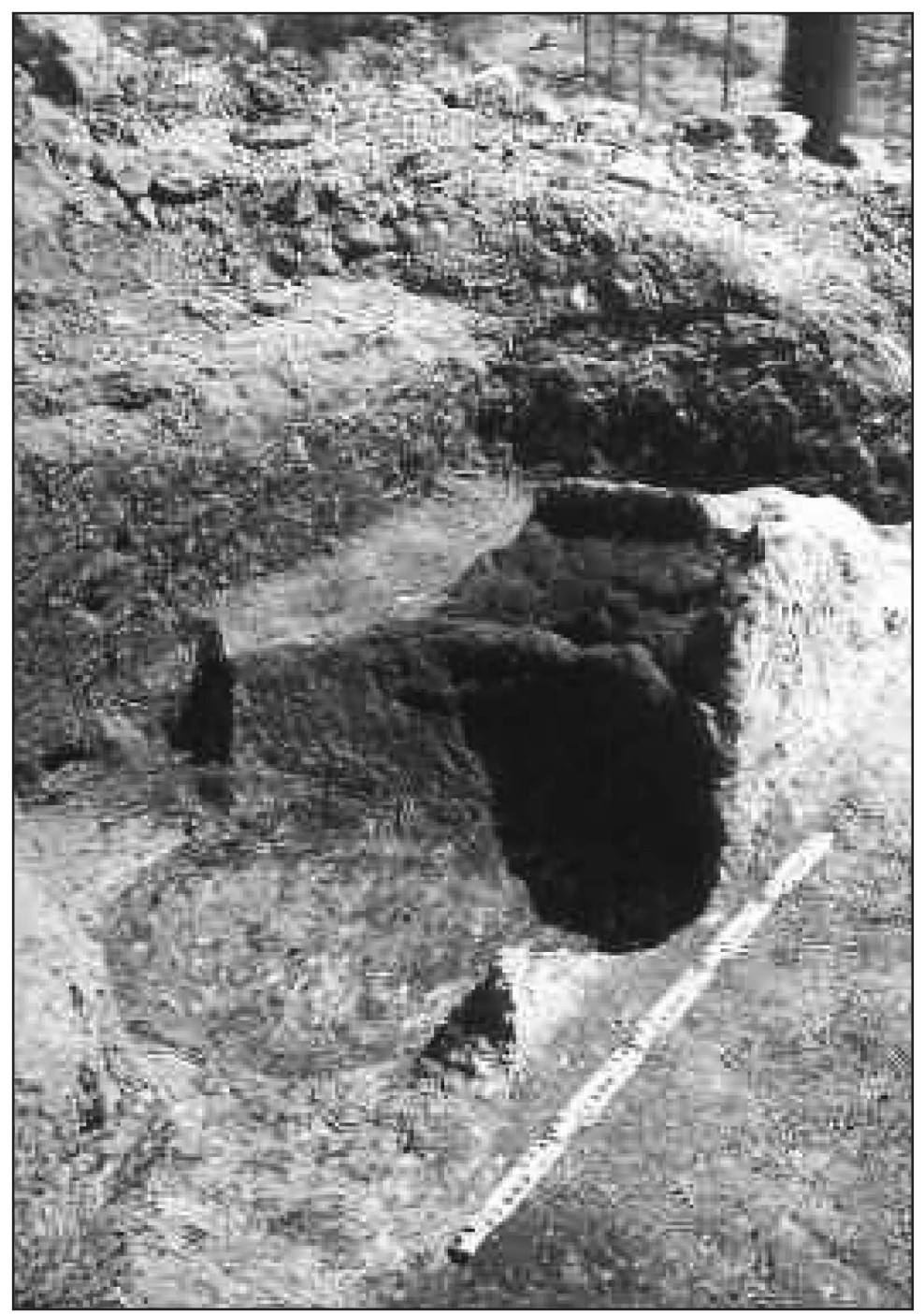

Figura 7. Posible horno de reducción del yacimiento ibérico de Les Guàrdies (El Vendrell, Tarragona) (Foto: http://www10.gencat.net/ agaur_web/recursos/La_Farga/Docs/1_3-.htm).

cubeta de forma hemi-esférica. Podemos reforzar esta referencia recurriendo a la ecuación de Bernoulli que, como hemos visto (vid. supra, 16), defiende que a más altura, más incremento térmico. Dubois recurrió a este mismo principio para sus interpretaciones experimentales con los hornos de Lercoul (Ariège) (Dubois, 2000 y 2001), que casi alcanzaban los dos metros de altura, y lo mismo puede decirse de los hornos experimentales de Les Martys (Aude) (Decombeix et alii, 2001), aunque en éstos casos se emplearon fuelles, discriminando a priori la posibilidad de que respondieran al principio del tiro libre. Siguiendo éstos ejemplos, podríamos tener en Les Guàrdies hornos de una altura similar, aproximadamente de 1'5-2 m.

Para inyectar mecánicamente aire en estas estructuras se requeriría un importante esfuerzo de naturaleza humana que manipule los fuelles (mínimo dos operadores por horno) y de un observador que controle la carga del horno durante los prolongados periodos de tiempo (entre 9 y 12 horas que pueden llegar a durar estas actividades). No obstante, debe tenerse en cuenta 
que tras el proceso de depuración en la fragua, una lupia de ese peso puede quedar reducida a unos cuantos gramos de hierro (vid. supra, 51).

Para aumentar estos índices de productividad de metal férrico, del todo insuficientes para su inmersión en circuitos comerciales, las opciones son multiplicar los hornos (con el consiguiente incremento en recursos humanos y la reducción de la rentabilidad) o la ampliación de la capacidad volumétrica de la cubeta. Como ya hemos comentado, alimentar manualmente un horno de tales dimensiones supondría, necesariamente, un más que notable incremento del esfuerzo y del tiempo necesarios.

En cambio, los hornos de alimentación libre pueden responder perfectamente a índices de productividad más elevados, disminuyendo considerablemente los recursos humanos necesarios y aumentando, por tanto, su "rentabilidad". En este sentido, los experimentos que hemos realizado, nos permiten afirmar que este tipo de hornos pueden ser considerados más ergonómicos y productivos, dado que una o dos personas podrían mantener en funcionamiento simultáneo un elevado número de ellos, cargándolos y abriéndolos, obteniendo de cada uno una gran cantidad de lupias que podrían ser depuradas y, quizás, transformadas en lingotes sobre grandes yunques pétreos in situ, para su posterior inserción en las redes comerciales. No pretendemos, con esto, descartar la posibilidad de que eventualmente se recurriera a la alimentación asistida de aire para acelerar el proceso de reducción ${ }^{11}$, cuya efectividad ha podido ser demostrada ya experimentalmente en diversas ocasiones (Sarabia, 1994; Boonstra, 1997), simplemente, proponemos la existencia, en el mundo ibérico, de explotaciones en las que el mineral fuera transformado a pie de mina. Existen, en este sentido, tres claros ejemplos. Se trata de los yacimientos ibérico-cosetanos de Les Guàrdies (El Vendrell, Tarragona) (Morer y Rigo 1999) y Mas d'en Gual (El Vendrell, Tarragona) (López, 2011) y de La Codina (Olius, Barcelona) (Cardona, 1999), centros de explotación agrícola y minera en los que se producía hierro, al menos durante la fase ocupada entre los ss. IV-III a. C. Entre los restos se encontraron evidencias de hábitat, minas a cielo abierto y numerosas estructuras de combustión, algunas de las cuales podrían tratarse de hornos de reducción directa y fraguas. Cabe destacar la significativa ausencia en el asentamiento de Les Guàrdies de restos de toberas y de piezas de insuflación de cerámica bífidas prefabricadas como las documentadas en otros yacimientos protohistóricos en los que se ha podido demostrar actividad metalúrgica, evidencia que, a nuestro juicio, apunta hacia la utilización de hornos de reducción directa de tiro libre. En cambio, en el caso de Mas d'en Gual, se recuperó una pieza bífida de insuflación que nosotros asociamos a la presencia en el núcleo de diversas fraguas.

Además, existe la suficiente documentación arqueológica para afirmar que en determinadas zonas mineras se llevó a cabo una explotación a cielo abierto de afloramientos ferríticos. Es el caso de las explotaciones mineras de óxidos (hematita y goethita) e hidróxidos de hierro (limonita) en les Ferreres de Gavà (Barcelona) (Álvarez y Estrada 2009, Bordas et alii, 2009), Valdestrada (Senó, Teruel) (Martín et alii, 2003), la Codina (Pinell, Barcelona) (Cardona et alii, 1999) y el Puig Castellar (Sant Vicenç dels Horts, Barcelona) (Rovira y Solías, 1991). La presencia de escorias de reducción de mineral en los yacimientos ibéricos del Calamot (Gavà, Barcelona) (Rovira, 2000: 215), del Puig Castellar (Sant Vicenç dels Horts, Barcelona) (Rovira y Solías, 1991), la Torre dels Encantats (Arenys de Mar, Barcelona) (Morell, 2008: 115), la

11 Tanto las lupias como las escorias de reducción de hierro recuperadas en contextos ibéricos presentan una composición esponjosa heterogénea semi-fundida, señal de un proceso de reducción rudimentario. No se han recuperado en esos contextos, en cambio, escorias de sangrado como las documentadas en centros productores de época romana, evidencia, a nuestro juicio, de un cambio evolutivo en el método, que probablemente implicaría la utilización de fuelles durante una fase final del proceso reductor. Es nuestra intención realizar experimentaciones teniendo en cuenta esta variable para constatar esta hipótesis. 
Torre Roja (Caldes de Montbui, Barcelona), Ca n'Oliver (Cerdanyola del Vallès, Barcelona), la Bastida de les Alcusses (Moixent, Alicante) (Bonet y Vives, 2011), además de las probables de Vallipón (Castellote, Teruel), Monte Catma (La Ginebrosa, Teruel), Pico de la Ginebrosa (La Ginebrosa, Teruel), Santa Flora (Mas de las Matas, Teruel) y los Cabanes (Mas de las Matas, Teruel) (todas ellas citadas en Martín et alii, 2003: 261) entre muchas otras, significaría que una de las actividades económicas desarrolladas en estos núcleos era la producción de hierro ${ }^{12}$. Desgraciadamente, no se puede defender la asociación de estas evidencias a una ocupación en hábitat, como en el caso de Les Guàrdies o Mas d'en Gual, dado que los escasos trabajos de excavación arqueológica realizados no permiten profundizar mucho más. No obstante, la presencia en otros lugares de Europa de asentamientos dedicados a la explotación intesiva de minerales férricos y a la producción de hierro puede servir como referente y paralelo, en este caso. Efectivamente, escenarios de diferente cronología, como los conjuntos de Les Martys (Decombeix et alii, 2000), Clérimois (Dunikowsky y Caboï, 1995) o Lercoul (Dubois, 2000) en Francia, los conjuntos romano-imperiales de Hoolbeanwood (Cleere, 1971) y de Laxton (Crew, 1998) en el Reino Unido, o los restos de múltiples hornos romanos bajo imperiales pendientes de excavación en el Bosc de Virós, en la Vall Ferrera (Pallars Sobirà) (Foret et alii, 2003) en España, demuestran la perduración a lo largo de diferentes periodos históricos de aquellos procesos y técnicas productivos rentables que funcionan correctamente, transmitidos y perpetuados a través de los diferentes grupos socio-culturales que se suceden en el tiempo.

La presencia de toberas de "tipo empuritano"13 y de piezas de insuflación prefabricadas bífidas, del "tipo ibérico" (fig. 8), según la clasificación propuesta por N. Molist (Molist et alii, 2004), en algunos espacios arqueológicos interpretados como talleres metalúrgicos, podría ser considerada, a priori, como un indicio de su empleo en hornos de reducción. Durante el proceso de documentación previo a la experimentación, encontramos en el registro arqueológico ibérico y celtibérico la existencia de un considerable número de piezas bífidas de "tipo ibérico". Se trata de las piezas del Castellet de Banyoles (Tivissa, Tarragona), Mas d'en Gual (El Vendrell, Tarragona), Olèrdola (S. Miquel d'Olèrdola, Barcelona), Olius (S. Esteve d'Olius, Barcelona), El Camp de les Lloses (Tona, Barcelona), La Bastida de les Alcusses (Moixent, Valencia), Piuró del Barranc Fondo (Mazaleón, Teruel), La Monravana (Llíria, Valencia), Cerro de San Cristóbal (Sinarcas, Valencia) y las dos piezas procedentes de Muela de Arriba (Requena, Valencia) $)^{14}$, además del ejemplar de Numancia (Garray, Soria) manufacturado a torno y erróneamente considerado en un principio como una trompa sonora, y del procedente de la Campa Torres (Gijón, Asturias). En los yacimientos de la Neápolis de Empúries (L’Escala, Girona) y el Puig de Sant Andreu (Ullastret, Girona), en cambio, se encuentran varios ejemplares de toberas de "tipo emporitano" (Ruiz de Arbulo, 1989).

12 Mostramos aquí nuestro total escepticismo ante la documentación en Valdestrada (Senó, Teruel) de un supuesto horno de reducción directa (Martín et alii, 2003) cuyas dimensiones excenden, a todas luces, los paralelos documentados en otros yacimientos ibéricos coetáneos y posteriores, y también la lógica. Los autores del artículo plantean la existencia en las immediaciones de la zona minera MDT II de Valdestrada de un horno de reducción semi excavado en la roca con unas dimensiones de 2'10 m. de diámetro y de 1’20 m. de altura conservados. La analítica publicada del fragmento de escoria encontrado en el interior de esta estructura muestra un 7\% de contenido en hierro (Fe) y más de un $50 \%$ de contenido en Dióxido de Sílice $\left(\mathrm{SiO}_{2}\right)$, elemento éste utilizado hoy en día para la producción de cementos en base de cal y muy abundante en restos de paredes de hornos escorificados que han estado sometidos a altas temperaturas (Rovira, 2000: 286, fig. 3). Estaríamos pues, a nuestro juicio, ante la parte excavada de un horno para producir cal mediante la cocción de la piedra caliza, tan abundante en la zona, dato que ellos mismos señalan.

13 Se trata de toberas con conducto único, de tipo corniforme, interpretadas como utensilios domésticos empleados en la cocina o como conductos de aire para trabajos de metalurgia (Ruiz de Arbulo, 1989).

14 Aunque no es descartable la existencia de una tercera pieza cuyos fragmentos, aún inéditos, se encuentran aún en fase de estudio (Mata, com. pers.). 


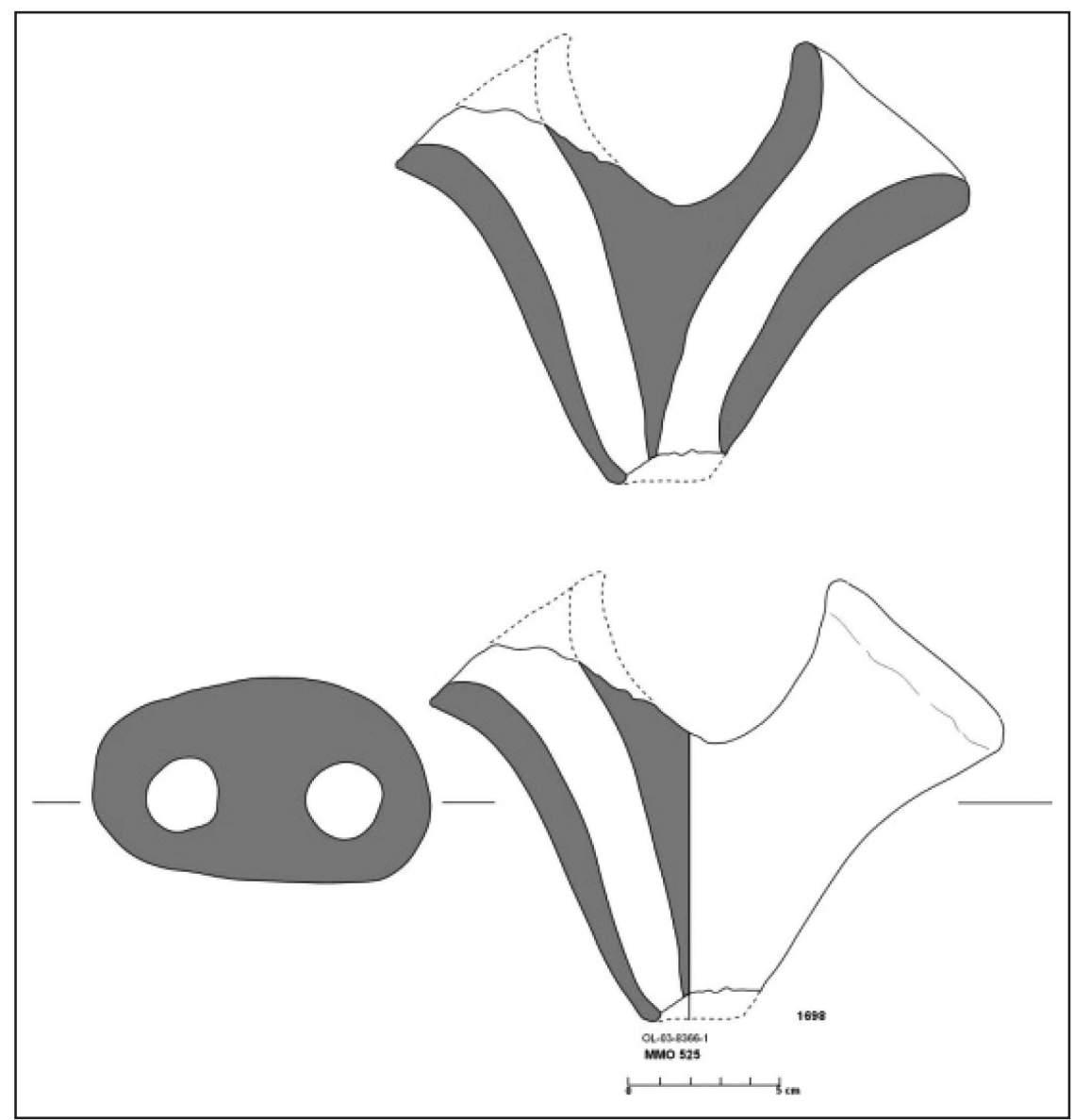

Figura 8. Sección de la tobera bífida encontrada en los niveles ibéricos en Olèrdola y datada en el s. III a. C. (Dibujo: Nuria Molist).

Tal como tuvimos oportunidad de comprobar empíricamente durante la experimentación con los hornos asistidos mediante fuelles (Gallego, en prensa), las piezas bífidas funcionan perfectamente como válvulas anti-absorción de aire caliente y de brasas del interior del horno gracias a su diseño. Pensadas para acoplarse a dos fuelles que funcionarían en ciclos alternativos, si observamos su sección advertiremos en seguida la existencia de dos canales que desembocan en uno solo ${ }^{15}$. Esta peculiaridad morfológica provoca que el aire sólo pueda circular en una dirección mientras se mantiene el flujo. La explicación es bien sencilla: los fuelles no pueden tomar aire del interior del horno a través de la pieza de insuflación bífida porque en cada ciclo de inyección ${ }^{16}$, uno de los canales se llena de aire fluyendo en dirección al horno, y llena a su vez, la tobera simple, cerrando así el circuito. El siguiente ciclo de inyección cierra el otro canal, y así sucesivamente. La escasa alteración térmica que se observa en el vértice de estas piezas (la parte que debería quedar integrada en el horno) es fácilmente justificable

15 Aunque la mayoría de ejemplares conservan el vértice fragmentado, siendo imposible decidir si convergían en un único tubo, la pieza del Cerro de San Cristóbal es especialmente ilustrativa dado que conserva este extremo intacto, definido por un único orificio de salida (Iranzo, 2004).

16 Este sistema de fuelles funciona perfectamente si alternamos las insuflaciones. Si los hacemos funcionar al mismo tiempo, se absorbe aire caliente y brasas del interior del horno. 


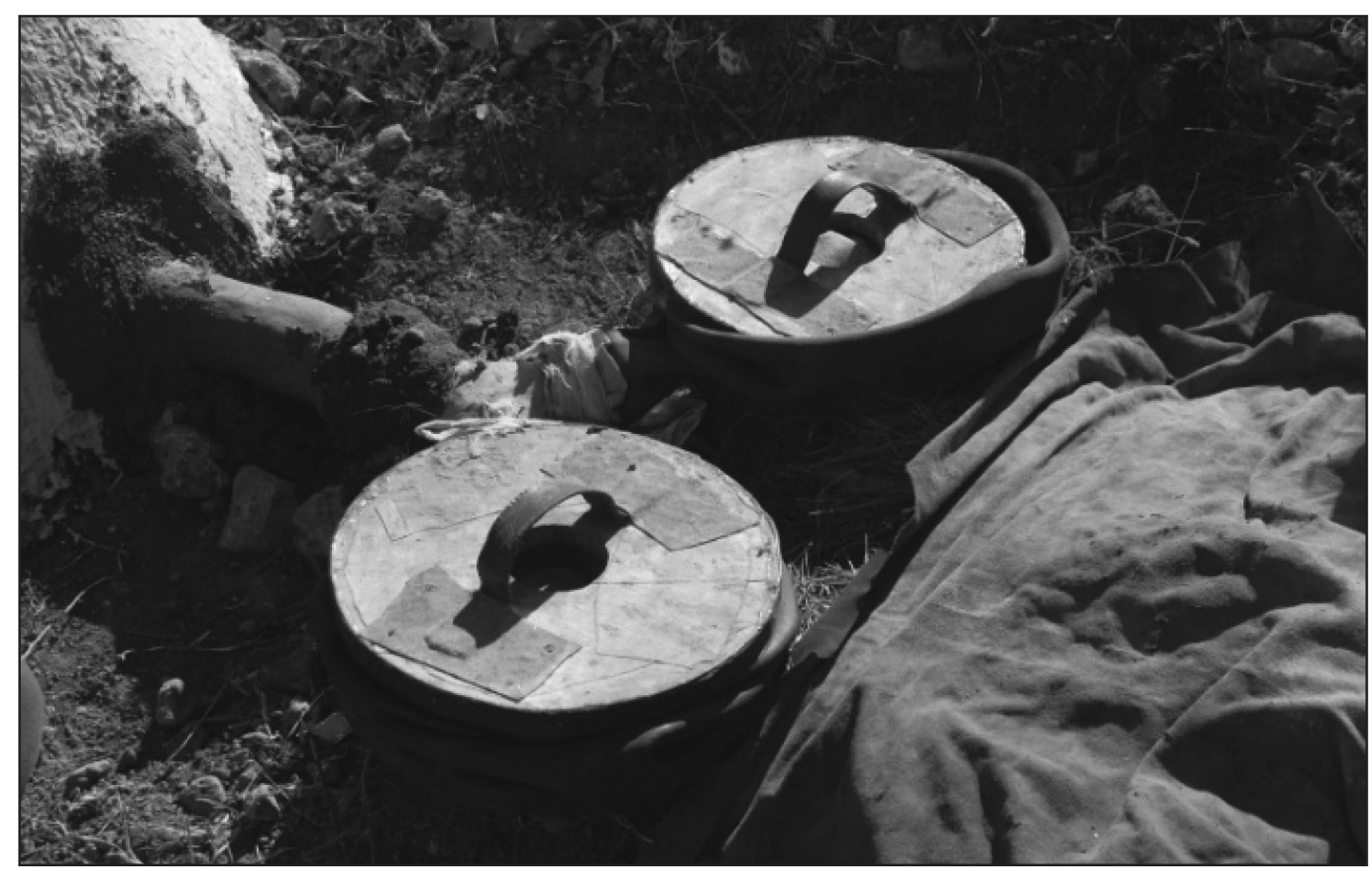

Figura 9. Imagen del sistema de inyección asistida para el horno de reducción "ELKAR", construido en Olèrdola para una demostración pública. Se puede apreciar claramente la disposición de los fuelles, de la pieza de inyección bífida y de la tobera cilíndrica (Foto: ARTIFEX, crpa)

si tenemos presente que, en realidad, este vértice quedaría, bien en el exterior del orificio de alimentación, bien acoplado a una tobera cilíndrica sencilla, que sería a su vez introducida en el horno (fig. 9).

La experimentación con dos hornos diferentes en los que empleamos dos piezas bífidas diferentes, reconstruidas por nosotros mismos, nos permitió advertir rápidamente los condicionantes del sistema de reducción mediante inyección manual de aire, los cuales hemos expuesto en las líneas superiores (vid. supra, 50-51 y cuya utilización en hornos de reducción nos plantea algunas dudas, debido, como hemos visto, a la gran inversión en recursos humanos que se necesitan para inyectar el aire necesario. Aunque no descartamos totalmente su empleo en pequeños hornos de reducción, dado que se trata de un elemento plenamente funcional y adecuado, en la actualidad concebimos más adecuada su utilización en trabajos de fragua. Nos apoyamos para afirmarlo, por un lado, en los argumentos que hemos expuesto con anterioridad respecto a la rentabilidad de hornos de reducción con alimentación mecánica. Por otro lado, estas piezas han aparecido en espacios urbanos identificados como talleres metalúrgicos, asociadas en general a pequeñas estructuras de combustión, que con toda probabilidad se correspondan a fraguas (vid. supra, 53-54). En ningún caso pudieron ser asociadas a hornos de reducción por sus excavadores. Finalmente, cabe señalar como dato curioso, que en la iconografía romana de cambio de Era, las piezas bífidas aparecen insertadas en el sistema de "fuelles de pistón", asociadas a pequeños talleres o fraguas de herrero (fig. 10).

Lo mismo podemos afirmar de algunas fraguas tradicionales centro-africanas en las que se empleaban unos sistemas de fuelles bífidos como recurso habitual (Maquet, 1965) (fig. 11). 


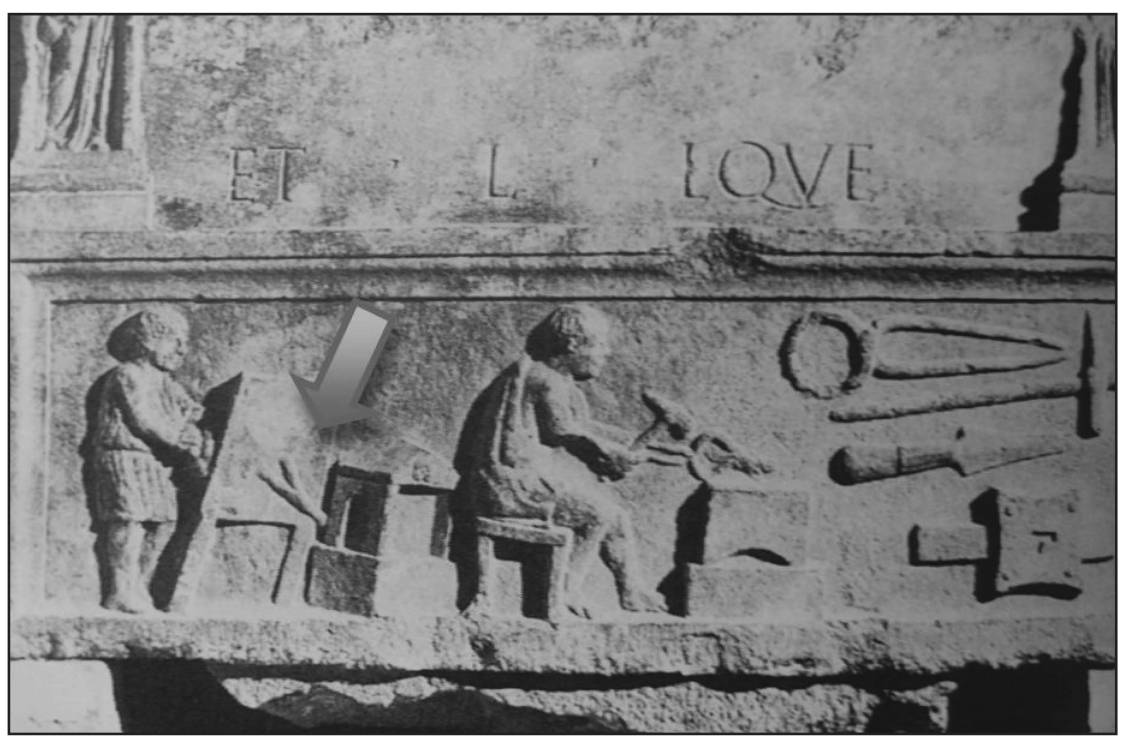

Figura 10. Relieve de una fragua romana (tomada de Ridge, I., Sim, D., 2002: 54) procedente del Museo de Aquileia. La flecha indica la presencia de la pieza bífida a la izquierda de la imagen.

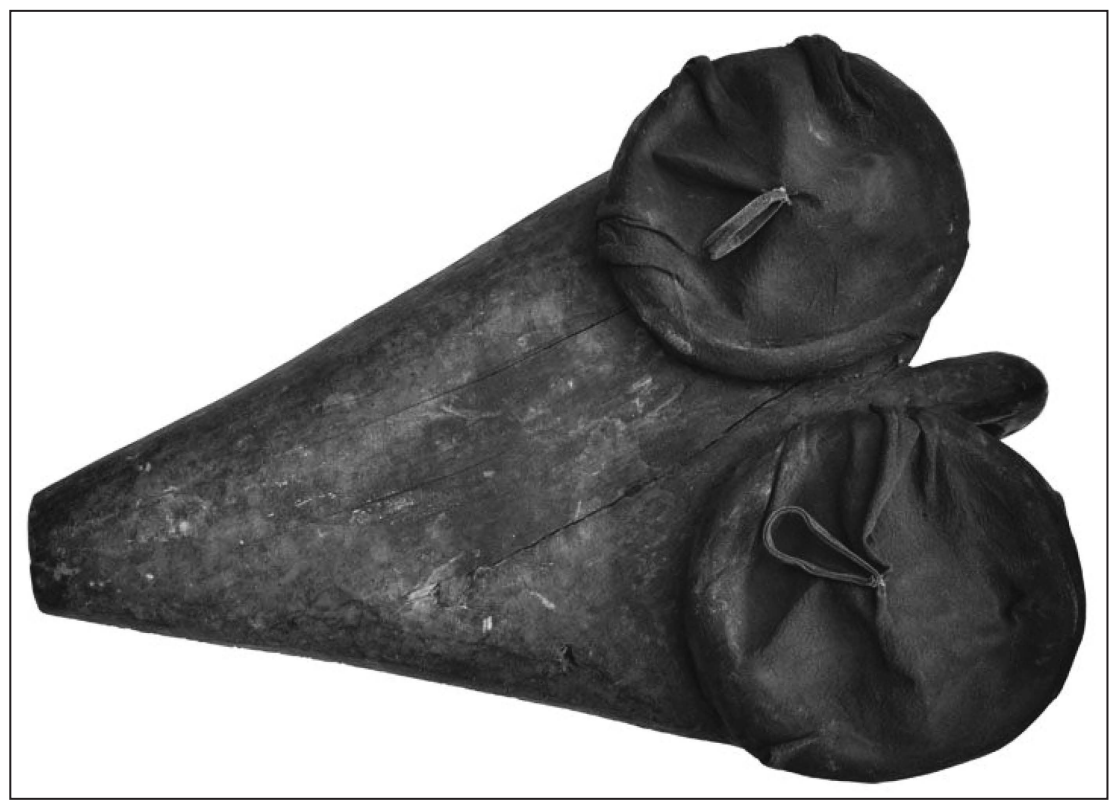

Figura 11. Sistema bífido de fuelles empleado en los trabajos de forja tradicional en Mali.

\section{CONCLUSIÓN}

A nuestro juicio, la presencia en el registro arqueológico ibérico de toberas bífidas debe asociarse a pequeñas fraguas destinadas quizá a la fabricación o reparación de herramientas o 
utensilios domésticos o, como mucho, a hornos de reducción de productividad muy limitada, siempre de carácter puntual y en ningún caso a centros de producción intensiva de hierro, para los que consideramos mucho más adecuados y rentables los hornos que utilizan el sistema de alimentación natural o de tiro libre.

Posibles hornos de reducción destinados a usos puntuales se han podido documentar en diversos yacimientos protohistóricos del territorio peninsular, siendo el ejemplo de Segeda (Burillo-Rovira, 2005) uno de los casos más evidentes (fig. 12), por hallarse en su interior un fragmento de lupia esponjosa, aunque también existen otros ejemplares en el Castellar

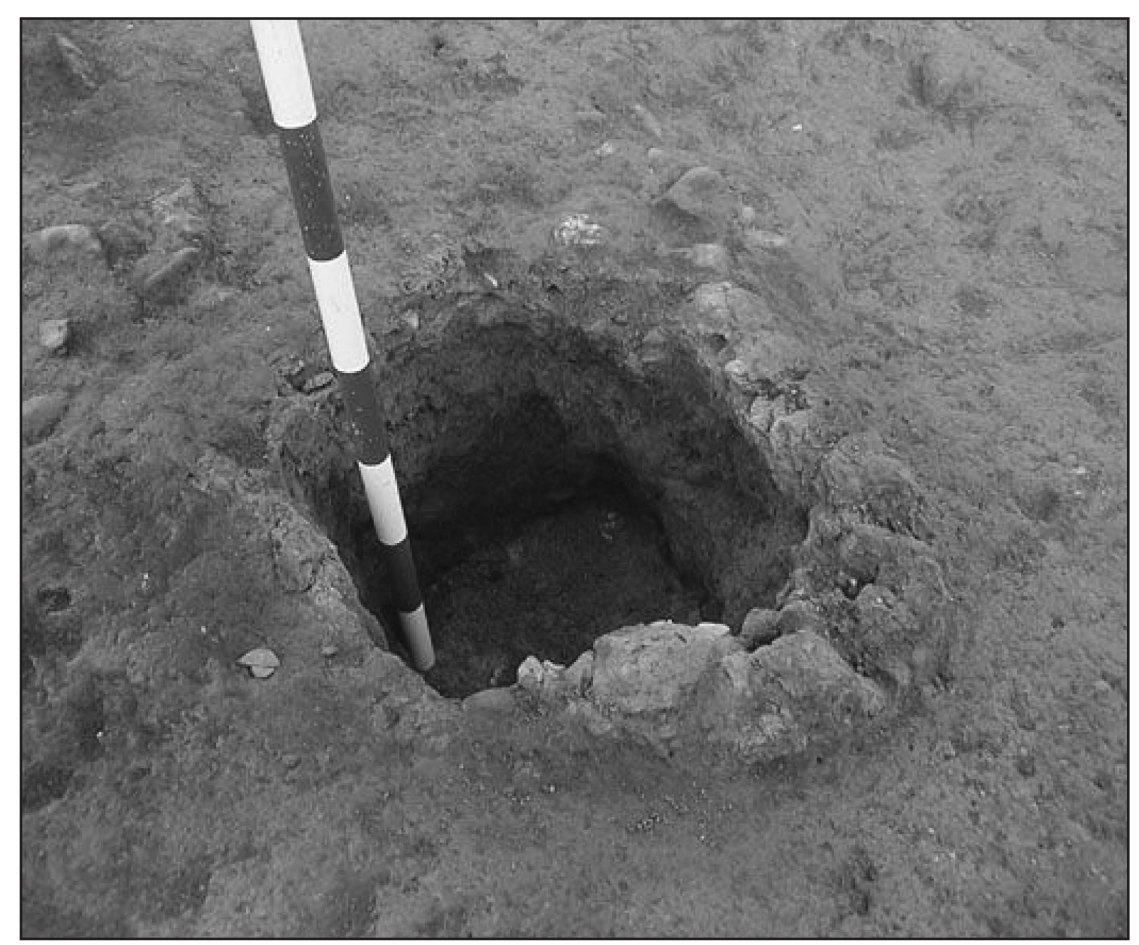

Figura 12. Restos de la cubeta para escorias y de la estructura aérea del horno de reducción directa de Segeda (Mara, Zaragoza) (Foto: F. Burillo).

(Librilla, Murcia) (Rovira, 2000: 267), la Bastida de les Alcusses (Moixent, Valencia) (Bonet y Vives, 2011) u Olèrdola (Sant Miquel d'Olèdola, Barcelona) (Molist, 2008). La verdadera producción con vocación comercial de hierro durante época ibérica, a nuestro juicio, debemos vincularla a explotaciones a pie de mina, eventualmente asociadas a hábitats, como en el caso de los yacimientos de Les Guàrdies, La Codina o Mas d'en Gual, datados a caballo entre el s. IV - III a. C., o en las explotaciones de la Sierra Menera (Teruel - Guadalajara) datadas entre el s. II a. C. y el s. II d. C. (Fabré et alii, 2012), donde la abundancia de materias primas favorecería una gestión más dinámica e intensiva, quizá con carácter estacional, concebida para transformar las esponjas de metal en lingotes susceptibles de ser integrados posteriormente en los circuitos comerciales del mundo ibérico (Ferrer, 2002: 200-201, Lorrio et alii, 1998-1999: 149), tan necesitados de este metal a partir del s. IV a. C., momento en el cual, y gracias a su perdurabilidad y versatilidad, se produce su generalización como material para la elaboración 
de herramientas agrícolas (Morer y Rigo, 1999: 30) y el incremento de su importancia como materia prima para la elaboración de armas en sociedades con una concepción de la fabricación y uso de armas arcaica en las que su posesión implicaba la ostentación de un estatus social determinado (Quesada, 2010: 37). Ello implicaría, obviamente, que desconocemos aún la mayor parte de la fase inicial de la producción de hierro durante la Protohistoria de la Península Ibérica. Sería necesario, por tanto, asumir como ineludible la investigación arqueológica en zonas tradicionalmente asociadas a explotaciones mineras en superfície para poder acercarnos más al conocimiento de este aspecto artesanal de las culturas ibéricas. Algunos autores proponen, además, una posible proximidad de centros productores de hierro a centros de producción alfarera dado que el combustible vegetal y los recursos hidrográficos constituyen elementos fundamentales para su desarrollo (Mata et alii, 2009).

Paralelamente, la existencia de explotaciones intensivas en las immediaciones de zonas mineras implicaría que no sería necesario transportar a las zonas pobladas las ingentes cantidades de materia prima (mineral, madera o carbón) necesarias para obtener hierro de forma intensiva. Pensamos que se transportarían las esponjas obtenidas en los hornos a pie de mina, probablemente ya depuradas y/ o lingoteadas. No obstante, tampoco podemos descartar la primera posibilidad: en los ejemplos franceses de Oulches (Indre- et- Loire) y de determinados talleres de Plateau de Haye (Lorraine central), se ha podido demostrar que el mineral fue transportado a una distancia de entre 10 y $20 \mathrm{~km}$. para su posterior tratamiento (Leroy y Merluzzo, 2004), aunque plausiblemente se trate de dos ejemplos excepcionales.

Por otro lado, la existencia de asentamientos vinculados a explotaciones intensivas de hierro, significaría, de la misma forma que ocurre con los asentamientos destinados al almacenaje del excedente agrario (Olius, Pontós, Avinyonet del Penedès, etc...), la existencia de una red de asentamientos cuya actividad económica se centra principalmente en la obtención de hierro y en las labores de la tierra, dependientes en gran medida del intercambio de hierro por otros alimentos y otros tipos de productos y elementos de uso cotidiano (Les Guàrdies, Mas d'en Gual). Es decir, se trataría de asentamientos no autosufucientes (Morer et alii, 1997: 86), sometidos a una jerarquización y ordenación territorial, controlada por clases sociales destacadas. No creemos que los encargados de su gestión fueran herreros convencionales, aunque tampoco podemos negar su conocimiento sobre aspectos básicos del trabajo de forja. Nos inclinamos a pensar en la existencia de grupos de personas especializadas y encargadas del desarrollo in situ del complejo proceso de elaboración del hierro (explotación minera, tratamiento del mineral, tala de vegetación, preparación de las carboneras, etc) cuyo objetivo es producir metal como medio complementario de subsistencia, razonamiento que Pleiner ya planteó, definiendo estos grupos como familiares (Pleiner, 2000). El primer paso en la elaboración de herramientas bélicas de hierro (y también de las herramientas agrícolas) durante época ibérica estaría, por tanto, en manos de grupos sociales dedicados exclusivamente a la obtención y primera modificación de la materia prima y no de su transformación final. A lo sumo, pensamos que estos especialistas podrían encargarse de la prepararación de lingotes de hierro para su posterior comercialización, orientada hacia los diferentes tipos de talleres de herrería, como los ampliamente documentados en diferentes yacimientos protohistóricos, algunos de los cuales han sido mencionados anteriormente (vid. supra, 23). Ello podría explicar la presencia de lingotes de hierro en yacimientos en los que no se ha documentado la presencia de hornos de reducción, como en La Bastida de les Alcusses (Bonet y Vives, 2011), Kelin (Mata et alii, 2009) o en el pecio de Les Sorres (Izquierdo, 1992: 21-25). Sería en estos talleres donde los maestros herreros especialistas administrarían los lingotes adquiridos para fabricar determinadas armas, utensilios y herramientas. Es sabido que los maestros herreros íberos conocían plenamente los aspectos relacionados con el tratamiento y trabajo del hierro a pesar de no poseer la pericia de otros pueblos coetáneos (Simon et alii, 2005). 
La existencia de éstos centros productores primarios de hierro podría responder a una tradición iniciada ya durante los siglos anteriores al s. VII a. C., con la explotación del cobre y la galena. Los restos evidenciados en algunos yacimientos del sur de Catalunya, como en el caso de Sant Jaume-Mas d'en Serra (Armada et alii, 2005b), apuntan hacia la existencia en toda la zona de una serie de poblados dedicados a la explotación intensiva de recursos mineros durante el Bronce Final, que se intensifica y diversifica a partir de la consolidación de las redes comerciales con elementos fenicios durante la I Edad del Hierro. El desarrollo socio-económico que experimentan las sociedades ibéricas a partir del s. IV a. C., se apoya firmemente en la generalización del hierro como materia prima para la elaboración de nuevas herramientas necesarias para la deforestación de zonas boscosas y los trabajos agrícolas y ello requiere una intensa producción de este metal; el incremento de la presencia en las necrópolis ibéricas de ajuares férricos a partir del s. IV a. C. (Quesada, 2002 y Quesada, 2010: 37), quizá constituye otra evidencia acerca de la necesidad de incrementar la producción de hierro respecto a las fases anteriores. Todo ello motivó, quizá, la génesis de nuevos asentamientos dedicados a una explotación intensiva de este metal tan polivalente, práctico y reversible durante los s. IV y III a. C, de los que Les Guàrdies, La Codina y Mas d'en Gual son los casos más evidentes.

Creemos que se hace evidente, ahora más que nunca, la necesidad de continuar con la investigación de campo combinada sistemáticamente con la arqueología experimental y los análisis arqueometalúrgicos con la intención de llegar a conclusiones más determinantes tanto en lo relativo a los procesos primarios de obtención de hierro como a los estrictamente ligados con el proceso de elaboración de armas.

\section{AGRADECIMIENTOS}

Quisieramos dedicar el presente trabajo a Magí Ballart Valls, familiar muy allegado tristemente fallecido mientras se redactaba el presente artículo. Hombre de gran bondad e integridad humana y dotado de una mente despierta, fue siempre promotor del conocimiento y la ciencia entre los suyos, y será siempre un ejemplo a seguir para los que tuvimos el placer y el honor de conocerle. Nuestros más sinceros respetos, Avi.

En el desarrollo de este trabajo de investigación he recibido la ayuda de diferentes personas. Especial agradecimiento merecen José Gallego, mi padre, siempre dispuesto a arrimar el hombro con paternal devoción y bajo cualquier circunstancia, como siempre, y Aida Alarcos, mi compañera, por su incondicional apoyo y paciencia, cualidades sin las que no se habrían podido llevar a cabo las experimentaciones que aquí se describen. También agradezco a Magí Ballart Alarcos todos los esfuerzos que dedicó para hacer entender a un "hombre de letras" los principios físicos de la dinámica de fluidos.

Asimismo quisiera agradecer a la familia Cantero (José, Dolores y Francesc) su constante paciencia y predisposición a cedernos el terreno, las herramientas y cualquier elemento logístico que hemos llegado a necesitar, que nunca han sido pocos; también agradecemos a Borja Gil, a Daniel Vázquez, a Pablo Martínez, a Walter A. Alegría, a Borja Tàpia, a Javier García, a Emiliano Hinojo y a Víctor Heredia todas las horas voluntarias que dedicaron a enfangarse, cortar y recolectar leña, remover estiércol de caballo, picar, cargar mineral en sus espaldas, en ocasiones bajo la lluvia, y el resto de tareas tan poco agradecidas que implica la experimentación siderúrgica.

Quisieramos agradecer también la ayuda recibida en la realización del presente artículo por parte de las siguientes personas, sin cuyas aportaciones y conocimientos habría resultado, a todas luces, mucho más difícil: Prof. Consuelo Mata (Universitat de València), Núria Molist 
(Museu d'Arqueologia de Catalunya), Prof. Salvador Rovira (Museo Arqueológico Nacional), Carme Rovira (Museu d'Arqueologia de Catalunya), Prof. Vincent Serneels (Fribourg University), Thijs Van de Mannaker (Eindhoven Museum), y Jaime Vives Ferrándiz (Museu de Prehistòria de València). Espero que en el futuro me permitan seguir incordiándoles.

\section{BIBLIOGRAFÍA}

Adelantado, P. (2003): "La metalurgia del hierro". En P. Guérin (ed.), El Castellet de Bernabé y el horizonte ibérico pleno edetano, Servicio de Investigación Prehistórica, Serie de Trabajos Varios: Diputación Provincial de Valencia, 101, 314-324.

Álvarez, R y Estrada, A. (2009): "L'explotació de ferro en el complex miner de Gavà. La mina n. ${ }^{\circ}$ 65", en L'arqueologia a Gavà, homentage a Alícia Estrada. Colección "La nostra gent", n. ${ }^{\circ}$ 5, 141160.

Andrieux, Ph. (2004): "Expérimenter en archéologie”. En M. Mangin (dir.), Le Fer. París: ed. Errance. Collection "Archéologies", 10-11.

Andrieux, Ph. (2004): " Les traces thermiques sur les parois de four". En M. Mangin (dir.), Le Fer. París: ed. Errance. Collection "Archéologies", 70-71.

Andrieux, Ph.; Domergue, C.; Jarrier, C.; Pieraggi, B. y Tollon, F. (1994): “ La sidérurgie antique. Des fourneaux romains remis en fonctionnement dans l'Aude ", Archéologia, 301, 59-66.

Armada, X. L.; Hunt, M. A.; Tresserras, J. J.; Montero, I.; Rafel, N. y Ruiz de Arbulo, J. (2005): "Primeros datos arqueométricos sobre la metalurgia del poblado y necropolis del Calvari del Molar (Priorat, Tarragona)", Trabajos de Prehistoria, 62, 1, 139-155.

Armada, X. L.; Garcia, D.; Montero, I.; Moreno; Rafel, N. y Rovira, M. C. (2005): "Minería y metalurgia durante la I Edad del Hierro. Procesos de cambio en el sur de Catalunya", Revista d'Arqueologia de Ponent (RAP). Lérida: Universitat de Lleida, . $^{\circ} 15,133-150$.

Auladell, J. (1993): "Metal·lúrgia i útils fèrrics agrícoles I ramaders pre- romans a l'àrea Laietània", Gala, 2, 227-236.

Auladell, J. (2004): Tecnologia del treball del ferro al Nord- est peninsular en l'Ibèric Antic i Ple, Tesis Doctoral dirigida por F. Gràcia y J. Simon, defendida en octubre de 2004. Barcelona : Universidad de Barcelona.

Auladell, J.y Simon, J. (1997): “Anàlisi metal·logràfica d'elements fèrrics pre- romans: una aproximació als mètodes siderúrgics a la Laietània", Pyrenae, 28, 119-132.

Avner, S. H. (1996): Introducción a la metalurgia física. Mèxico, D. F.

Blakelock, E.; Martinón, M.; Veldhuijzen, H. A.y Young, T. (2009): "Slag inclusions in iron objects and the quest for provenance: an experiment and a case study", Journal of Archaeological Science, 36, 1745-1757.

Bonet, H.; Vives-Ferrándiz, J. (eds.) (2011): La Bastida de les Alcusses. 1928-2010. Valencia: Museu de Prehistòria de València, 119-124.

Boonstra, A.; Van de Manakker, T. y Van Dijk, W. (1997): "Experiments with a slag- tapping and a slag pit furnace". En Lars Chr. Nørbach (ed.), Early iron production. Archaeology, technology and experiments. Nordic Iron Seminar. Lejre, del 22 al 28 de julio, 1996, Technical Report, 1997, n. ${ }^{\circ}$ 3, 73-80.

Bordas, A.; Molinas, R.; Saa, M. (2009): "La mineria del ferro a Gavà. Darreres intervencions arqueològiques a la serra de les Ferreres". En L'arqueologia a Gavà, homentage a Alícia Estrada, colección "La nostra gent", n. ${ }^{\circ}$ 5, 161-176.

Burillo, F. y Rovira, S. (2005): "Experimentos de fundición de minerales de hierro en la ciudad-estado celtibérica de Segeda (Mara, Zaragoza)". En Avances en Arqueometría. Girona: Universitat de Girona, 137-145.

Cardona, R., (1999): "Memòria arqueològica del jaciment del Camp dels Moros de la Codina, Pinell, Campanya 1999". Servei de Patrimoni arqueològic i paleontològic del Departament de Cultura de la Generalitat de Catalunya.

Chapa, T. y Mayoral, V. (2007): Arqueología del trabajo. El ciclo de la vida en un poblado ibérico. Madrid: ed. Akal, Akal Arqueología, 90-101. 
Cleere, H. F. (1971): "Ironmaking in a Roman furnace", Britannia, 2, 203-217.

Cleere, H. F. (1972): "The classification of early smelting-iron furnaces", The Antiquaries Journal II, $8-23$.

Craddock, P. T. y Meeks, N. D. (1983-1988): "Iron in copper". En G. Speri (ed.), The first iron in the Mediterranean, Populonia-Piombino, 1983, PACT, 21, Strasbourg, 1988, 119-130.

Crew, P. (1991): “The Experimental Production of Prehistoric Bar Iron”, Historical Metallurgy 25, 1, 21-36.

Crew, P. (1998): "Laxton revisited: a first report on the 1998 excavations", Historical Metallurgy, 32, 2, 49-53.

Decombeix, P.; Domergue, C.; Fabré, J. M.; Gorgues, A.; Tollon, F. y Tournier, B. (2000): "Reflections sur l'organisation de la production du fer a l'époque romaine dans le bassin supérieur de la Dure, au voisinage des Martys (Aude)", Gallia, 23-36.

Decombeix, P. M.; Fabré, J. M. y Rico, Ch. (2001): "Expérimentations de réduction directe du fer dans des reconstitutions des bas-fourneaux romains de la Montagne Noire". En Actes del ler Simposi sobre la Mineria i la Metal-lúrgia antiga al sud-oest europeu.Centre d'Arqueologia d'Avinganya, Seròs, 5 al 7 de mayo del 2000, 185-194.

Domergue, C. y Leroy, M. (2000): “L'etat de la recherche sur les mines et les métallurgies en Gaule, de l'époque gauloise au haut Moyen Âge. En C. Domergue"; M. Leroy (eds.), Mines et métallurgies en Gaule, Gallia, 57, 3-10.

Dubois, C. (2000): "Lercoul (Pyrénées ariégeoises): une site sidérurgique du IIIè s. de notre ère". En C. Domergue y M. Leroy (eds.), Mines et métallurgies en Gaule, Gallia, 57, 53-62.

Dubois, C. (2001): "Elements sur l'origine de la production du fer en Ariège: le site de Lercoul, en L'obtenció del ferro pel procediment directe entre els segles IV i XIXS", Actes del Gè curs d'Arqueologia d'Andorra. Andorra.

Dunikowsky, M. y Cabboï, S. (1995): "La sidérurgie chez les Sénons: les ateliers celtiques et gallo- romains des Clérimois (Yonne)", Documents d'Archéologie Françoise, 51. Paris: Maison des Sciences de l'Homme.

Ehren, Th.; Charlton, M.; Chirikure, S.; Humphris, J.; Ige, A. y Veldhuijzen, H. A. (2007): "Decisions Set in Slag. The Human Factor in African Iron Smelting". En S. la Niece; D. R. Hook; P. T. Craddock (eds.), Metals and Mines. Studies in Archaeometallurgy. London: Archetype, British Museum, 211-218.

Espelund, A. (2010): "Experimental ironmaking once more: combining theory and find material", EuroREA, 7, 4-8.

Fabré, J. M.; Polo, C.; Rico, Ch. y Villargordo, C. (2012): "Minería y siderurgia Antigua en Sierra Menera (Teruel-Guadalajara). Nuevos avances de la explotación del hierro en época Antigua (ss. II a. C.-II d. C.)". En Minería y Metalurgia antiguas. Visiones y revisiones. Madrid: Casa de Velázquez, 43-62.

Ferrer, M. A. (2000): "La metalurgia ibérica del hierro. Una aproximación a través de la interpretación arqueométrica". En C. Mata, y G. Pérez, (eds.), Ibers. Agricultors, artesans i comerciants. III Reunió sobre Economia en el Món Ibèric, SAGVNTUM Extra - 3, Universitat de València, 283-290.

Ferrer, M.A. (2002): "La actividad extractiva y metalúrgicas. En H. Bonet y C. Mata (eds.), El Puntal dels Llops. Un fortín edetano, Servicio de Investigación Prehistórica, Serie de Trabajos Varios, 99: Diputación Provincial de Valencia, 192-211.

Forbes, R. J. (1971): Studies in Ancient Technology, VIII, Leiden. $2^{\mathrm{a}}$ edición revisada.

Forbes, R.J. (1972): Studies in Ancient Technology, IX, Leiden. $2^{\mathrm{a}}$ edición revisada.

Forés, A.; Gassiot, E. y Oltra, J. (2003): "Mes enllà de la transhumància: mineria i metal·lúrgia antiga a l'alta muntanya del Pallars Sobirà". En J. M. Mata-Perelló (ed.). Libro de Actas del Primer Simposio sobre Minería, Metalurgia y Patrimonio en el Área Mediterránea, Bellmunt del Priorat, del 8 al 10 de Noviembre del 2002: Ajuntament de Bellmunt, SEDPGYM, UPC, 71-82.

Foster, J. (1995): "Metalworking in the British Iron Age". En A. Raftery; V. Megaw y V. Rigby (eds.), Sites and Sights of the Iron Age. Essays on fieldwork and Museum Research presented to Ian Mathieson Stead. Oxford, 49-60.

Gallego, J. M. (2013): "La siderurgia en el mundo ibérico. Primeros datos a partir de la experimentación arqueológica". Actas del III Congreso Internacional de Arqueología Experimental, Banyoles, del 17 al 19 de octubre de 2011. Barcelona: Museu d'Arqueologia de Catalunya. 
Garcia, G. (2006): "Entre íberos y celtas. Las espadas de tipo "La Tène" del Noreste de la Península Ibérica", Anejos de Gladius, 10. Madrid: CSIC, Instituto Histórico Hoffmeyer. Instituto de Historia. Ediciones Polifemo.

García, G. (2012): El armamento de influencia. La Têne en la Península Ibérica, Monographies Instrumentum. Montagnae, 43.

Gómez Ramos, P. (1996): “Análisis de escorias férreas: nuevas aportaciones al conocimiento de la siderurgia prerromana en España”, Trabajos de Prehistoria, 53, vol. 2, 1996, 145-155.

Iranzo Viana, P. (2004): “Arqueología e Historia de Sinarcas", Ayuntamiento de Sinarcas, Valencia.

Izquierdo, P. (1992): "El jaciment arqueològic de les Sorres". En Les Sorres X. Un vaixell medieval al canal olímpic de rem, Castelldefels, Baix Llobregat, Memòries d'intervencions arqueològiques a Catalunya, 1, 21-25.

Karbowniczek, M.; Weker, W.; Suliga, I. (2009): "Experimental metallurgical process in a slag pit bloomery furnace", EuroREA 6, 45-49.

Leroy, M. y Merluzzo, P. (2004): “La réduction: du minerai au métal”. En M. Mangin (dir.), Le Fer, ed. Errance, Collection "Archéologies". París, 49-80.

López, D. (2011) : "Memòria científica de l'excavació arqueològica a l'assentament ibèric (segles III- II a. C.) de Mas d'en Gual (el Vendrell, Tarragona)". Servei de Patrimoni arqueològic i paleontològic del Departament de Cultura de la Generalitat de Catalunya.

Lorrio, A. J.; Rovira, S., y Gago, F. (1998-1999): "Una falcata damasquinada procedente de la Plana de Utiel (Valencia)", Lucentum, XVII-XVIII, Alicante, 149-161.

Lorrio, A.; Gómez, P.; Montero, I. y Rovira, S. (1999): "Minería y metalurgia celtibérica”. En IV Simposio sobre Celtiberos. Economía. Zaragoza, 161-180.

Madroñero de la Cal, Á. (1989): "Los hierros de la España prerromana”. En C. Domergue (ed.), Minería y metalurgia en las antiguas civilizaciones mediterráneas y europeas, Ministerio de Cultura, 109118.

Mangin, M. (dir.) (2004): Le fer, Col. Archéologiques. Paris: Errance.

Mangin, M. y Fluzin, PH. (2006): "L'organisation de la production métallurgique dans une ville galloromaine: le travail du fer à Alésia", Revue archéologique de l'Est 55, 129-150.

Manning, W. H. (1995): "Ironworking in the Celtic World". En The Celtic World. Londres : 310-320.

Maquet, E. (1965) : .Outils de forge du Congo, du Rwanda et du Burundi. Tervuren : Musée Royal de l'Afrique centrale.

Martín, A.; López, V. y Gabaldón, A. (2003): "El conjunto minero- metalúrgico del término municipal de Senó (Bajo Aragón): un ejemplo del origen y la difusión de la siderurgia protohistórica en el levante español", Salduie, 3, 257-267.

Martinelli, B. (2008) : "Patrimoine sidérurgique traditionnel en Centrafrique". En Revue Centre-Africaine d'Anthropologie, ${ }^{\circ} 1$. La métallurgie du fer en Centrafrique, (on line).

Mata, C.; Moreno, A. y Ferrer, M. A. (2009): "Iron, Fuel and Slags: reconstructing the Ironworking Process in Iberian Iron Age (Valencian Region)", Pyrenae, 40, vol. II, 105-127.

Mohen, J. P. (1992): Metalurgia prehistórica. Introducción a la paleometalurgia. Barcelona.

Molist, N.; Ros, A.; Bosch, J. M. ${ }^{\text {a; }}$ Mestres, J. y Senabre, M. ${ }^{a}$ R. (2005): "Piezas de insuflación de aire del proceso metalúrgico en el noreste peninsular durante el período ibérico". En O. Puche y M. Ayarzagüena (eds.). Actas del II Simposio sobre minería y metalurgia históricas en el sudoeste europeo, Madrid, 24-26 de junio de 2004. Madrid, 179-188.

Molist, N. (ed.) (2008): "La intervenció al sector 01 del Conjunt Històric d'Olèrdola. De la Prehistòria a l'etapa romana (campanyes 1995-2006)", Monografies d'Olèrdola, 2, Museu d'Arqueologia de Catalunya, 175-182.

Morell, N. (2008): "Treballant el ferro: canvis i continuitats al nord- est de la Península Ibèrica durant els segles II-I a. C.". En M. Miñarro y S. Valenzuela (eds.), Actes del I Congreso de Joves Investigadors en Arqueologia dels Països Catalans: la protohistoria als Països Catalans, Arqueomediterrània, 10, 113-122.

Morer, J.; Rigo, A. y Barrasetas, E. (1997): “Les intervencions arqueològiques l'autopista A- 16: valoració de conjunt", Tribuna d'Arqueologia, 1996-1997, 67-98.

Morer, J. y Rigo, A. (1999): Ferro i ferrers en el món ibèric. El poblat de les Guàrdies (El Vendrell): ed. Aucat, Autopistes de Catalunya. 
Morer, J. y Rigo, A. (2003): Les Guàdies (El Vendrell, Baix Penedès). Un assentament metal lurque d'època ibérica en Territoris antics a la Mediterrànea i a la Losseetànica oriental, el Vendrell, Actes del Simposi Internacional d'Arqueología del Baix Penedès, del 8 al 10 de noviembre de 2001, Departamento de Cultura, Generalitat de Catalunya, 327-338.

Oliver, A (1996): "Poblamiento y territorio protohistóricos en el llano litoral del Baix Maestrat (Castellón)". Castellón: Sociedad Castellonense de Cultura, Arqueologia-IX.

Panareda, J. M. y Masnou, J. (2010): El carboneig. Quan el record encara és viu, Publicacions de l'Abadia de Montserrat.

Pelet, P. L. (1993): "Une industrie reconnue: Fer, Charbon, Acier dans le Pays de Vaud", Cahiers d'Archéologie Romande, 60, Lausanne.

Pleiner, R. (1980): Early iron Metallurgy in Europe. , London: Ed. Wertin Muhly.

Pleiner, R. (1988): "Les primeres produccions de ferro a l'Europa Central i Oriental", Cota Zero, 13, Vic, $71-84$.

Quesada, F. (1997): El armamento ibérico. Estudio tipológico, geográfico, funcional, social y simbólico de las armas en la Cultura Ibérica (siglos VI-I a. C.), Monographies Instrumentum, 3. Montagnac.

Quesada, F. (2002): "La evolución de la panoplia, modos de combate y tácticas de los iberos", en $L a$ guerra en el mundo ibérico y celtibérico (ss. VI-VII a. C.). Collection de la Casa de Velázquez, vol. 78. Madrid, 35-64.

Quesada, F. (2010): "Las armas en los poblados ibéricos. Teoría, método y resultados", Gladius, XXX, 17-42. Madrid: CSIC.

Quesada, F.; Gabaldón, M.; Requena, F. y Zamora, M. (2000): “¿Artesanos itinerantes en el mundo ibérico? Sobre técnicas y estilos decorativos, especialistas y territorio". En C. Mata y G. Pérez (eds.), Ibers. Agricultors, artesans i comerciants. III Reunió sobre Economia en el Món Ibèric, SAGVNTUM Extra- 3. Valencia: Universitat de València, 291- 301.

Rehder, J. E. (2000): The mastery and uses of fire in Antiquity. Montreal (Canadá): McGill-Queen's University Press Montreal.

Reynolds, P. J. (1988): Arqueologia experimental. Una perspectiva de futu. Vic: Eumo. Col. Referències, 4.

Rostocker, W. y Bronson, B. (1990): "Pre-industrial iron, its technology and ethnology", Archaeomaterials Monograph, 1. Philadelphia.

Rovira, M. C. (1997): "De bronzistes a ferrers: dinàmica de la metal·lúrgia protohistòrica al nord-est peninsular", Cota Zero, 13, 59-70.

Rovira, M. C. (2000): "Los talleres de herrero en el mundo ibérico. Aspectos técnicos y sociales; en C. Mata, C. y G. Pérez (eds.), Ibers. Agricultors, artesans i comerciants. III Reunió sobre Economia en el Món Ibèric, SAGVNTUM Extra-3. Valencia: Universitat de València, 265-270.

Rovira, M. C. (2012): "La producció siderurgica en època ibèrica a Catalunya". En Boscos de Ferro, Actes de les Primeres Jornades de Recerca i Desenvolupament de la Vall Ferrera. Tremp: Garsineu Edicions, 41-50.

Rovira, S.; Burillo; F; López, R. e Ibáñez, J. (2012): "Metalurgia y explotación de recursos minerales en el entorno de la ciudad-estado celtibérica de Segeda I (Mara, Zaragoza)". En A. Orejas y C. Rico (eds.), Minería y metalurgia antiguas. Visiones y revisiones. Madrid: Casa de Velázquez, 27-42.

Rovira, S. (1993): "La metalurgia de la Edad del Hierro en la Península Ibérica: una síntesis introductoria”. En R. Arana; A. M. Muñoz; S. Ramallo i Ros, M. ${ }^{a}$ M. (eds.), Metalurgia en la Península Ibérica durante el Primer Milenio a. C. Estado actual de la investigación. Murcia: Universidad de Murcia, 45-70.

Rovira, S. (2000): "Continuismo e innovación en la metalurgia ibérica”. En C. Mata, y G. Pérez (eds.), Ibers. Agricultors, artesans i comerciants. III Reunió sobre Economía en el MónIbèric Valencia, Universidad de Valencia, SAGVNTUM Extra-3, 209-221.

Rovira, S. y Solías, J. M. (1991): "Iron minery and metallurgy in the lower course of river Llobregat (N. E. of Spain) during the Roman Republican period", en International Archaeometallurgical Symposium", Materialy Archeologiczne, XXVI: 53-57.

Ruiz de Arbulo, J. (1989): "Soportes y toberas. Los ejemplares de Emporion", Archivo Español de Arqueología, 62, 315-324. 
Sarabia, F. J. (1994): “Aproximación teórica y metalográfica a la reducción de hierro en la Prehistoria partiendo del trabajo experimental", Trabajos de Prehistoria, 5, 95-109.

Schmidt, P. (ed.) (1996): The Culture and Technology of African Iron Production. Florida: University Press.

Serneels, V. (1997): "L’estudi dels rebutjos metal·lúrgics i la seva aportació a la comprensió de la industria del ferro", Revista Cota Zero, 13, 29-42.

Serneels, V. (1998): "La chaîne opératoire de la sidérurgie ancienne”. En M. Feugère y V. Serneels (dirs.), Recherches sur l'économie du fer en Méditérranée nord-occidentale. Montagnac: Monographie Instrumentum, 4, 7-44.

Serneels, V. (2004): "Le Fer et ses minerais". En M. Mangin (dir.), Le Fer. París: ed. Errance. Collection "Archéologies", 25-48.

Serneels, V. y Crew, P. (1997): “Ore-Slag Relationships from experimentally Smelted Bog-Iron Ore". En P. Crew y S. Crew (eds.), Early Ironworking in Europe, archaeology and experiment, international Conference at Plas Tan Y Bwlch, Plas Tan y Bwlch Occassional Paper, 3, 78-82.

Serneels, V. Huysecom, E. y Fluzin, Ph. (1997): “Inagina Iron- slags and eutectoïd steel”, Early Ironworking in Europe, archaeology and Experiment, International Conference Plas Tan y Bwlch, Plas Tan y Bwlch Ocassional paper.Sim, D. y Ridge, I. (2011): Iron for the eagles. The iron industry of Roman Britain. Gloucestershire: The History Press.

Simon, J.; Tartera, J.; Marsal, M. y Auladell, J. (2005): "De los íberos al imperio romano. Evolución tecnológica del hierro", Revista de Metalurgia, n. ${ }^{\circ}$ esp, 507-512. Madrid: CSIC.

Tylecote, R. F.; Austin, J. N. y Wraith, A. E. (1971): "The mechanism of the bloomery process in shaft furnaces", Journal Iron Steel Institute, 209, 342-363.

Urteaga, M. (1997): "Programa internacional de experimentaciones en la ferrería de Agorregui”, Arkeolan, 6 1/99, 11-13.

Veldhuijzen, H. A. y Rehren, Th. (2007): "Slags and the City. Early Iron Production at Tell Hammeh, Jordan, and Tel Beth-Shemesh, Israel". En S. la Niece; D. R. Hook and P. T. Craddock (eds.), Metals and Mines. Studies in Archaeometallurgy. London, Archetype, British Museum, 189-201.

Recibido: $29 / 02 / 2012$

Aceptado: 12/06/2014 